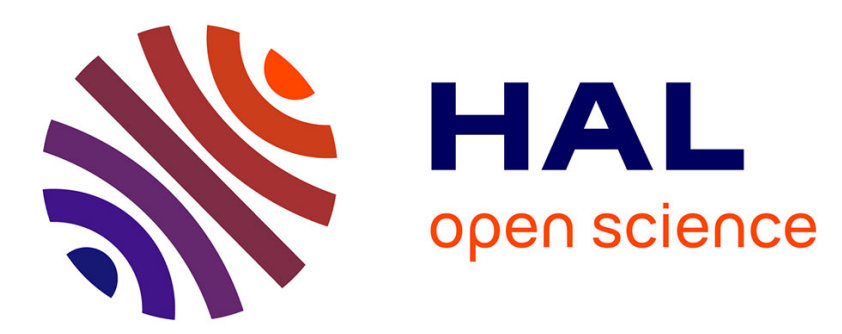

\title{
Vibration of cylindrical shells with embedded annular acoustic black holes using the Rayleigh-Ritz method with Gaussian basis functions
}

\author{
Jie Deng, Oriol Guasch, Laurent Maxit, Ling Zheng
}

\section{To cite this version:}

Jie Deng, Oriol Guasch, Laurent Maxit, Ling Zheng. Vibration of cylindrical shells with embedded annular acoustic black holes using the Rayleigh-Ritz method with Gaussian basis functions. Mechanical Systems and Signal Processing, 2021, 150, pp.107225. 10.1016/j.ymssp.2020.107225 . hal-02936140

\author{
HAL Id: hal-02936140 \\ https://hal.science/hal-02936140
}

Submitted on 11 Sep 2020

HAL is a multi-disciplinary open access archive for the deposit and dissemination of scientific research documents, whether they are published or not. The documents may come from teaching and research institutions in France or abroad, or from public or private research centers.
L'archive ouverte pluridisciplinaire HAL, est destinée au dépôt et à la diffusion de documents scientifiques de niveau recherche, publiés ou non, émanant des établissements d'enseignement et de recherche français ou étrangers, des laboratoires publics ou privés. 


\title{
Vibration of cylindrical shells with embedded annular acoustic black holes using the Rayleigh-Ritz method with Gaussian basis functions
}

\author{
Jie Denga ${ }^{\mathrm{a}, \mathrm{b}}$, Oriol Guasch ${ }^{\mathrm{b}, *}$, Laurent Maxit ${ }^{\mathrm{c}}$, Ling Zheng ${ }^{\mathrm{a}}$ \\ a State Key Laboratory of Mechanical Transmission, College of Automotive Engineering \\ Chongqing University, Chongqing 400044, PR China \\ ${ }^{b}$ GTM - Grup de Recerca en Tecnologies Mèdia, La Salle, Universitat Ramon Llull \\ C/ Quatre Camins 30, 08022 Barcelona, Catalonia (Spain) \\ ${ }^{c}$ INSA Lyon, Laboratoire Vibrations-Acoustique (LVA) \\ 25 bis, av. Jean Capelle, F-69621 Villeurbanne Cedex, France
}

\begin{abstract}
The numerical simulation of beams and plates with embedded acoustic black holes (ABHs) is computationally demanding because of the very thin thickness attained at the ABH central area. Semi-analytical approaches relying on the Rayleigh-Ritz method with wavelet or Gaussian basis functions have thus revealed as an accurate and fast alternative to determine the $\mathrm{ABH}$ vibration field in parametric studies. To date however, the vast majority of works on $\mathrm{ABH}$ have only dealt with $\mathrm{ABH}$ indentations on straight beams and flat plates. It would be also worth exploring the feasibility of ABHs to control the vibrations of curved shells, typically found in aerospace and naval structures. In this work, we address this issue and extend the Gaussian expansion method (GEM) to characterize annular ABHs embedded on cylindrical shells. First, we show how the GEM can be modified to make Gaussian shape functions satisfy periodic boundary conditions in the circumferential direction of the cylinder. The GEM is then used to determine the vibration field of the ABH cylindrical shell and gets validated by comparison with finite element simulations. A thorough analysis of the performance of the annular $\mathrm{ABH}$ follows, which stresses the differences with the behavior of $\mathrm{ABHs}$ on flat surfaces. In particular, we show the influence that waves propagating in the circumferential direction have on the operational frequency range of the ABH. The effects of the viscoelastic layer and the inclusion of longitudinal stiffeners to strengthen the cylinder rigidity are also analyzed by means of the proposed GEM approach. This work broadens previous semi-analytical methods to start investigating the ABH effect on curved structures.
\end{abstract}

Keywords: Annular acoustic black holes, Gaussian expansion method, Rayleigh-Ritz method, Cylindrical shells, Stiffened ABHs

\section{Introduction}

Acoustic black holes (ABHs) have drawn increasing attention in the past decade as a passive, lightweight and highly-efficient method for vibration [1-5] and noise [6-8] control, energy harvesting [9, 10] or focusing [11, 12], and wave manipulation [13-15]. With the possible exception of the Archimedean spiral $\mathrm{ABH}$ for beams that was investigated numerically in [16] and experimentally in [17], almost all works to date have dealt with $\mathrm{ABH}$ indentations on straight beams [18, 19] and flat plates [20-22]. However, many built-up engineering structures contain curved beams and shells. For instance, aircrafts, underwater vehicles or pipeline systems are a few examples in which cylindrical shells are the major structural components. It would be therefore worth exploring whereas ABHs could be designed and exploited to reduce the vibrations of curved shells. This paper proposes a semi-analytical model for an annular ABH embedded on a cylindrical

\footnotetext{
*Corresponding Author: oguasch@salle.url.edu
} 
shell and analyzes its performance. To date, only very recent numerical simulations have been carried out to tackle this problem $[23,24]$.

The ABH effect relies upon the fact that a flexural wave propagating in a structure whose thickness decays to zero following a power-law profile slows down in such a way that the energy concentrates at its tip, but never reaches the end $[25,26]$. In theory, this would imply that no reflection could occur from the ABH but in practice the small tip truncation thickness can result in strong wave reflection. Several strategies have been proposed to avoid that problem and attain large energy dissipation inside the ABH. The former comprise from placing a simple viscoelastic layer at the ABH tip [27, 28], a constrained one [19], or to extend the $\mathrm{ABH}$ ending platform [29,30], among other options. More recently, optimization algorithms have been suggested to properly distribute the damping material or ABH profile to reduce vibration [31-34] and sound radiation [35].

To reproduce the vibration field of an $\mathrm{ABH}$, we need to resort to semi-analytical or numerical approaches. Given that ABHs involve very thin structural elements, numerical models (e.g., a finite element model) often become costly from a computational point of view because very fine meshes are required. Semi-analytical methods then constitute a valuable alternative to perform parametric analyzes. As for the characterization of vibrations of flat structures, several approaches have been successfully contemplated in the ABH context. Geometrical acoustics, for instance, have been suggested for infinite plates [36, 37], the transfer matrix method has been used for beam [28] and duct ABH terminations [38-41], the WKB (Wentzel-KramersBrillouin) approximation has been applied to wedged beams [42] and even exact solutions for some special Euler-Bernoulli profiled beams have been derived [43]. In addition, a large amount of work has been performed in the framework of the Rayleigh-Ritz method: from initial attempts using trigonometric basis functions [44] to more recent wavelet proposals for straight beams [18, 19] and flat plates [20, 21]. Tested shape functions include Mexican hat wavelets [18], Daubechies scaling functions [20], Morlet wavelets [12] and Gaussian functions. The use of Gaussian basis functions in the Rayleigh-Ritz method will be hereafter referred to as the Gaussian expansion method (GEM) and was applied to beams and plates in [19, 21, 22].

None of the aforementioned methods has yet been exploited to deal with ABHs on curved shells. In this article the GEM will be adapted to this purpose. It is to be noted that trigonometric shape functions have been extensively and successfully used to reproduce the vibration field of uniform and rib reinforced cylinders [45, 46]. Unfortunately, thin cuneate ABH areas are very challenging and pose limits to the precision one could obtain with trigonometric functions [44]. This is the reason why the semi-analytical methods based on wavelet or Gaussian shape function decompositions are favored in the ABH literature. Our goal in this work is to extend the GEM to apply it to the annular ABHs embedded on cylindrical shells recently suggested in $[23,24]$. This implies modifying the Gaussian shape functions so that they fulfill periodicity in the cylinder circumferential direction. Closed expressions can be derived for the GEM and thus avoid, for example, the iterative process that is necessary for building a Mexican hat wavelet basis for the periodic $\mathrm{ABH}$ beam in [47]. It will be shown that the GEM provides an accurate and fast way to characterize annular ABHs on cylinders.

On the other hand, the GEM will be employed to investigate the functioning of the annular ABH. The latter presents some differences with regard to its analogues in flat plates and beams. For example, given that waves can propagate in the cylinder circumferential direction which cannot be mitigated by the annular $\mathrm{ABH}$, the standard diameter and smoothness cut-on frequencies that dictate the operational range of ABHs in flat surfaces do not straightforwardly apply to the present situation. Other aspects will be also explored, such as the inclusion of longitudinal stiffeners to compensate for stiffness weakening induced by the $\mathrm{ABH}$ indentation [24].

The paper is organized as follows. In Section 2 we state the problem to be solved, while in Section 3 we present the GEM for a cylindrical shell with an embedded annular ABH. The Lagrangian of the model is built and it is explained how to construct a proper basis of Gaussian functions to expand the displacement field and satisfying the problem periodic boundary conditions. The GEM approach is then validated against finite element (FEM) simulations in Section 4. The performance of the annular ABH is characterized in Section 5, where its operational frequency range is tested, as well as the influence of the size of the viscoelastic damping layer and the inclusion of structural stiffeners. Conclusions are drawn in Section 6. Two appendices are respectively devoted to present the mass and stiffness matrices of the system as well as to detail the 
expression for the GEM basis.

\section{Geometrical model}

Throughout this paper we will consider the case of a thin cylindrical shell of length $2 a$, radius $R$ and thickness $h_{u n i}$. The origin of coordinates is placed at the shell center. A three-dimensional sectional view of the geometry is plotted in Fig. 1a, which is complemented with a planar sectional cut in Fig. 1b. The cylindrical shell boundary conditions are accounted for by means of uniformly distributed springs. Assigning different values to the spring rotational stiffness, $p$, and translational stiffness, $k$, one can represent various boundary conditions. For instance, setting $p=+\infty$ and $k=+\infty$ we recover clamped boundary conditions, while $p=+\infty$ and $k=0$ results in simply supported ones (note in Fig. 1a that $p_{1}$ and $k_{1}$ stand for the springs' stiffness at the cylinder left end, whereas $p_{2}$ and $k_{2}$ refer to those in the right end).

An annular ABH is embedded in the cylindrical shell occupying the region [ $\left.-r_{\mathrm{abh}}, r_{\mathrm{abh}}\right]$. The thickness of the shell within the ABH diminishes according to the power law $h(x)=\varepsilon x^{m}+h_{c}(m \geq 2)$, where $\varepsilon=\left(h_{\mathrm{uni}}-h_{c}\right) r_{\mathrm{abh}}^{-m}$. The variable $h_{c}$ represents the residual thickness at the ABH center and plays a critical role to achieve a significant $\mathrm{ABH}$ effect. A thin viscoelastic layer of thickness $h_{v}$ and covering a length $2 r_{v}$ (green area in Fig. 1) is attached to the ABH to dissipate the flexural vibration energy that concentrates at its center. A cylindrical shell of constant thickness and having the same axial region than the $\mathrm{ABH}$ one covered by a damping layer will also be considered for comparison purposes (see Fig. 1).

In what follows, we will deal with the case of an external point force exerted at $x=x_{f}$ (see the red arrow in Fig. 1), which induces displacements, $u$, in the axis direction, $v$, in the circumferential one and $w$, in the radial direction.

\section{Gaussian expansion method for cylindrical shells with an annular ABH indentation}

\subsection{Equations of motion}

In this section, a semi-analytical method is proposed for obtaining the displacement field of a cylindrical shell with an embedded annular ABH. The equations of motion are herein derived in the framework of Rayleigh-Ritz method using Gaussian basis functions. As mentioned in the Introduction, we will refer to this approach as the GEM (Gaussian Expansion Method). The basis functions must be such that they satisfy the shell periodicity conditions in the circumferential direction.

A shell model is implemented with $x, y$ and $z$ respectively designating coordinates in the axial, circumferential and radial directions and $u, v$ and $w$ denoting the corresponding shell displacements (see Fig. 1a). The displacement components $(u, v, w)$ refer to the mid surface (the center of mass surface) and so does the radius $R$. According to Love's theory [48], the strain components at an arbitrary point of the cylindrical shell can be expressed as,

$$
\varepsilon_{x}=\varepsilon_{x_{0}}+z_{e} \kappa_{x}, \varepsilon_{y}=\varepsilon_{y_{0}}+z \kappa_{y}, \varepsilon_{x y}=\varepsilon_{x y_{0}}+z \kappa_{x y},
$$

where $z_{e}$ is the distance to the shell mid-surface,

$$
\varepsilon_{x_{0}}=\frac{\partial u}{\partial x}, \varepsilon_{y_{0}}=\frac{\partial v}{\partial y}+\frac{w}{R}, \varepsilon_{x y_{0}}=\frac{\partial v}{\partial x}+\frac{\partial u}{\partial y},
$$

stand for the mid-surface strain-displacement relations and

$$
\kappa_{x}=-\frac{\partial^{2} w}{\partial x^{2}}, \kappa_{y}=-\left(\frac{\partial^{2} w}{\partial y^{2}}-\frac{1}{R} \frac{\partial v}{\partial y}\right), \kappa_{x y}=-2\left(\frac{\partial^{2} w}{\partial x \partial y}-\frac{1}{R} \frac{\partial v}{\partial x}\right),
$$

account for the mid-surface changes in curvature and torsion. Neglecting the stress components $\sigma_{z}, \sigma_{x z}$ and $\sigma_{y z}$, the strain potential energy of the cylindrical shell can be calculated as

$$
U_{\text {shell }}=\frac{1}{2} \int_{-\pi R}^{\pi R} \int_{-a}^{a} \int_{-h(x) / 2}^{h(x) / 2} \boldsymbol{\sigma}^{\top} \varepsilon \mathrm{d} z \mathrm{~d} x \mathrm{~d} y,
$$



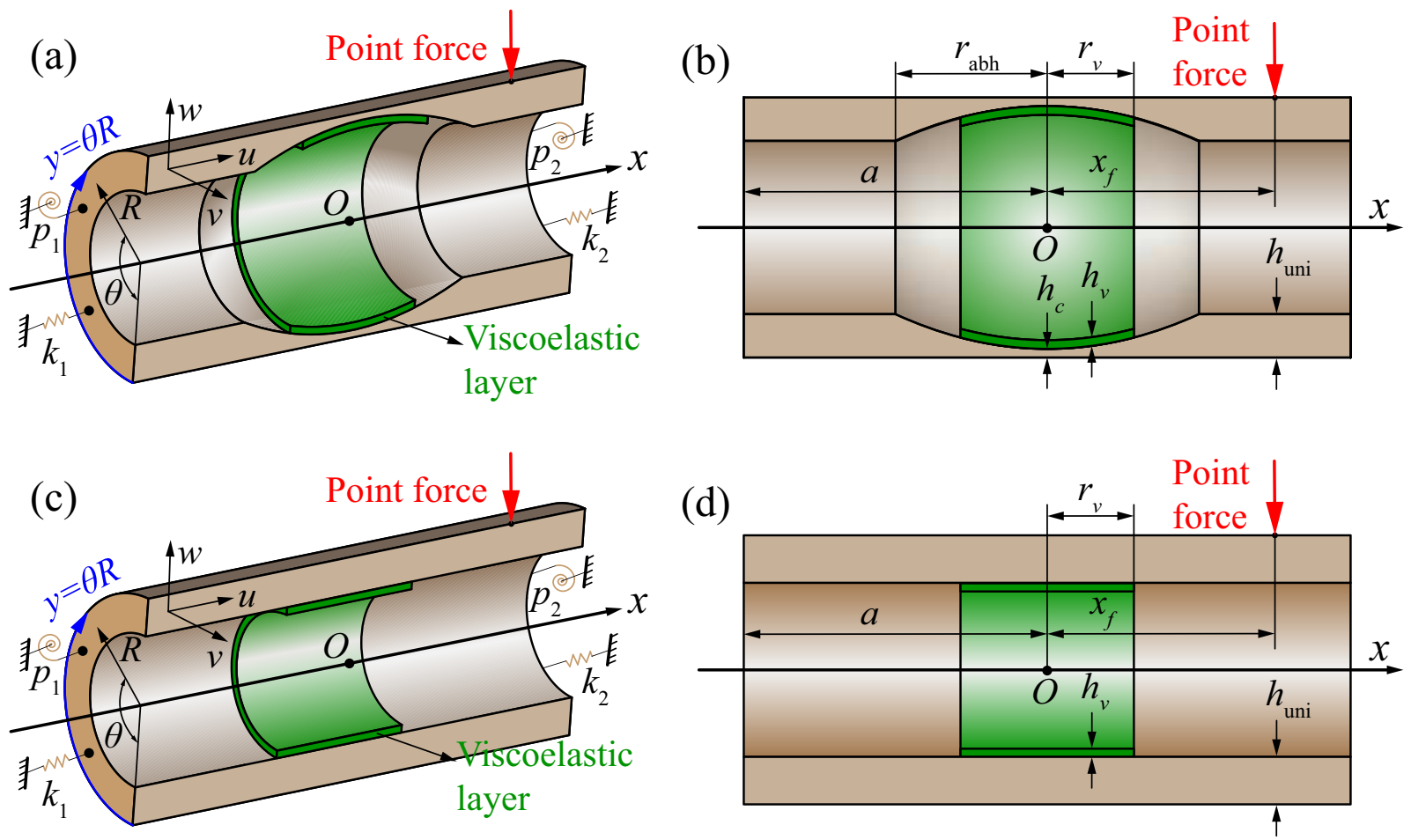

Figure 1: Illustration of the cylindrical shell with an embedded annular ABH profile. The green shaded area corresponds to the viscoelastic layer. (a) 3D section cut, (b) cross-sectional view. (c)-(d) uniform reference shell having the same damping layer as the ABH shell in (a)-(b). Note that the thickness of the shells has been exaggerated in the plots to better appreciate the geometrical details and definitions. In the considered examples the shell thickness is very thin in comparison to the shell radius $R$.

with stress, $\sigma$, and strain, $\varepsilon$, vectors

$$
\boldsymbol{\sigma}=\left(\sigma_{x}, \sigma_{y}, \sigma_{x y}\right)^{\top}, \boldsymbol{\varepsilon}=\left(\varepsilon_{x}, \varepsilon_{y}, \varepsilon_{x y}\right)^{\top} .
$$

The strain-stress relation is given by $\boldsymbol{\sigma}=\boldsymbol{E} \boldsymbol{\varepsilon}$, with $\boldsymbol{E}$ standing for the material constitutive matrix of the shell,

$$
\boldsymbol{E}=\frac{E^{\star}}{1-\nu^{2}}\left[\begin{array}{ccc}
1 & \nu & 0 \\
\nu & 1 & 0 \\
0 & 0 & \frac{1-\nu}{2}
\end{array}\right]
$$

in which $\nu$ represents the Poisson ratio and $E^{\star}=E(1+\mathrm{j} \eta)$ a complex Young modulus to allow the inclusion of damping through the material loss factor $\eta$. 
Combining Eqs. (1) to (6), the potential energy of the cylindrical shell can be rewritten as,

$$
\begin{aligned}
U_{\text {shell }}=\frac{1}{2} \int_{-\pi R}^{\pi R} \int_{-a}^{a} D(x, y) & {\left[\left(\frac{\partial^{2} w}{\partial x^{2}}\right)^{2}+\left(\frac{\partial^{2} w}{\partial y^{2}}-\frac{1}{R} \frac{\partial v}{\partial y}\right)^{2}\right.} \\
& \left.+2 \nu \frac{\partial^{2} w}{\partial x^{2}}\left(\frac{\partial^{2} w}{\partial y^{2}}-\frac{1}{R} \frac{\partial v}{\partial y}\right)+\frac{1-\nu}{2}\left(2 \frac{\partial^{2} w}{\partial x \partial y}-\frac{2}{R} \frac{\partial v}{\partial x}\right)^{2}\right] \mathrm{d} x \mathrm{~d} y \\
+\frac{1}{2} \int_{-\pi R}^{\pi R} \int_{-a}^{a} G(x, y)\left\{\left(\frac{\partial u}{\partial x}\right)^{2}+\left(\frac{\partial v}{\partial y}+\frac{w}{R}\right)^{2}\right. & \left.+\nu\left[\frac{\partial u}{\partial x}\left(\frac{\partial v}{\partial y}+\frac{w}{R}\right)+\left(\frac{\partial v}{\partial y}+\frac{w}{R}\right) \frac{\partial u}{\partial x}\right]+\frac{1-\nu}{2}\left(\frac{\partial v}{\partial x}+\frac{\partial u}{\partial y}\right)^{2}\right\} \mathrm{d} x \mathrm{~d} y
\end{aligned}
$$

where $D(x, y)=\frac{E^{\star} h^{3}(x, y)}{12\left(1-\nu^{2}\right)}$ is the bending stiffness and $G(x, y)=\frac{E^{\star} h(x, y)}{1-\nu^{2}}$ the extensional one.

Further, one must also account for the potential energy stored in the boundary springs, say $U_{\text {edge. This }}$ is given by,

$$
\begin{aligned}
U_{\text {edge }}= & \frac{1}{2} \int_{-\pi R}^{\pi R}\left\{k_{1}^{w} u^{2}(-a, y)+k_{1}^{v} v^{2}(-a, y)+k_{1}^{w} w^{2}(-a, y)+p_{1}^{w}\left[\frac{\partial w}{\partial x}(-a, y)\right]^{2}\right\} \mathrm{d} y \\
& +\frac{1}{2} \int_{-\pi R}^{\pi R}\left\{k_{2}^{u} u^{2}(a, y)+k_{2}^{v} v^{2}(a, y)+k_{2}^{w} w^{2}(a, y)+p_{2}^{w}\left[\frac{\partial w}{\partial x}(a, y)\right]^{2}\right\} \mathrm{d} y .
\end{aligned}
$$

On the other hand, the kinetic energy of the shell is obtained as

$$
K_{\text {shell }}=\frac{1}{2} \int_{-\pi R}^{\pi R} \int_{-a}^{a} \rho h(x, y)\left[\left(\frac{\partial u}{\partial t}\right)^{2}+\left(\frac{\partial v}{\partial t}\right)^{2}+\left(\frac{\partial w}{\partial t}\right)^{2}\right] \mathrm{d} x \mathrm{~d} y .
$$

In addition to the potential and kinetic energies of the shell, we have to consider those of the viscous damping layer as well. There are different ways to deal with the latter, see e.g., [18, 19, 44]. Among them, the fully coupled method [18] is widely adopted as it has proved fairly accurate when comparing theoretical predictions with experiments (see e.g., $[18,49]$ ). The method treats the damping layer as an additional system layer with its intrinsic material properties so that its potential energy, $U_{\text {vis }}$, and kinetic energy, $K_{\text {vis }}$, can be respectively computed from Eq. (7) and Eq. (9), by simply replacing the geometrical and material properties of the shell with those of the viscous damping layer.

Finally, we need to take into account the work done by the external forces. In this paper, only point forces in the radial direction will be contemplated, so we get

$$
W=f(t) w\left(x_{f}, y_{f}, t\right) .
$$

Note that more complex force distributions could be also examined quite easily. For instance, the external work done by a ring force applied at the axial coordinate $x_{f}$ would be given by $W=f(t) \int_{-\pi R}^{\pi R} w\left(x_{f}, y, t\right) \mathrm{d} y$.

From all the above expressions, we are now in disposition of building the Lagrangian as,

$$
\mathscr{L}=K_{\text {shell }}+K_{\text {vis }}-\left(U_{\text {shell }}+U_{\text {edge }}+U_{\text {vis }}\right)+W .
$$

To derive the equations of motion, however, let us first discretize the system by expressing the displacements $u, v, w$ as a combination of basis functions $\psi_{i}(x, y), \xi_{i}(x, y), \varphi_{i}(x, y)$ (to be specified below), with a priori unknown weight coefficients $a_{i}(t), b_{i}(t), c_{i}(t)$. Namely,

$$
u(x, y, t)=\sum_{i} a_{i}(t) \psi_{i}(x, y)=\boldsymbol{a}^{\top} \boldsymbol{\psi}=\boldsymbol{\psi}^{\top} \boldsymbol{a}
$$




$$
\begin{gathered}
v(x, y, t)=\sum_{i} b_{i}(t) \xi_{i}(x, y)=\boldsymbol{b}^{\top} \boldsymbol{\xi}=\boldsymbol{\xi}^{\top} \boldsymbol{b} \\
w(x, y, t)=\sum_{i} c_{i}(t) \varphi_{i}(x, y)=\boldsymbol{c}^{\top} \boldsymbol{\varphi}=\boldsymbol{\varphi}^{\top} \boldsymbol{c}
\end{gathered}
$$

where $\boldsymbol{a}, \boldsymbol{b}, \boldsymbol{c}, \boldsymbol{\psi}, \boldsymbol{\xi}$ and $\boldsymbol{\varphi}$ are column vectors with entries $a_{i}(t), b_{i}(t), c_{i}(t), \psi_{i}(x, y), \xi_{i}(x, y)$ and $\varphi_{i}(x, y)$, respectively. With the aid of Kronecker's product, the vectors $\boldsymbol{\psi}, \boldsymbol{\xi}$ and $\boldsymbol{\varphi}$ can be factored as

$$
\begin{gathered}
\boldsymbol{\psi}(x, y)=\boldsymbol{\alpha}^{\psi}(x) \otimes \boldsymbol{\beta}^{\psi}(y), \\
\boldsymbol{\xi}(x, y)=\boldsymbol{\alpha}^{\xi}(x) \otimes \boldsymbol{\beta}^{\xi}(y), \\
\boldsymbol{\varphi}(x, y)=\boldsymbol{\alpha}^{\varphi}(x) \otimes \boldsymbol{\beta}^{\varphi}(y),
\end{gathered}
$$

where $\boldsymbol{\alpha}^{k}, k=\psi, \xi, \varphi$, is a column vector containing basis functions that only depend on the $x$ direction. Likewise, $\boldsymbol{\beta}^{k}, k=\psi, \xi, \varphi$, represents a column vector of basis functions in the $y$ direction.[50] Our choice has been to select Gaussian functions for the basis, so the entries $\alpha_{i}^{k}(x)$ become

$$
\alpha_{i}^{k}(x)=2^{s_{x} / 2} \exp \left[-\left(2^{s_{x}} x-q_{x_{i}}\right)^{2} / 2\right],
$$

which consist of a translation plus binary dilation of the Gaussian function $g(x)=\exp \left(-x^{2} / 2\right)$. The ranges of the scaling parameter $s_{x}$ and translation parameter $q_{x}$ are taken as

$$
\begin{gathered}
s_{x} \geq \delta_{x}, \delta_{x}=\text { ceil }\left[\log _{2}\left(\frac{8}{2 a}\right)\right], \\
q_{x}=\left[-4+\text { floor }\left(-2^{s_{x}} a\right), \operatorname{ceil}\left(2^{s_{x}} a\right)+4\right],
\end{gathered}
$$

where ceil $(x)$ stands for the largest integer smaller than $x$ and floor $(x)$ for the smallest integer greater than $x$. Analogous expressions to Eqs. (18)-(20) can be build for the functions in the y-direction, $\beta_{i}^{k}(y)$. The reader is referred to the authors previous work in $[19,21]$ for further details on the selection of the scaling and translation ranges for the Gaussian functions.

With the above decompositions for the displacement field, the Lagrangian in Eq. (11) can be rewritten as

$$
\mathscr{L}=\frac{1}{2} \dot{\boldsymbol{q}}^{\top} \boldsymbol{M} \dot{\boldsymbol{q}}-\frac{1}{2} \boldsymbol{q}^{\top} \boldsymbol{K} \boldsymbol{q}+\boldsymbol{q}^{\top} \boldsymbol{f}
$$

where $\boldsymbol{q}=\hat{\boldsymbol{Q}} \exp (\mathrm{j} \omega t)$ accounts for the time dependent vector of unknowns with amplitude $\hat{\boldsymbol{Q}}=\left[\hat{\boldsymbol{A}}^{\top}, \hat{\boldsymbol{B}}^{\top}, \hat{\boldsymbol{C}}^{\top}\right]^{\top}$, because $\boldsymbol{a}=\hat{\boldsymbol{A}} \exp (\mathrm{j} \omega t), \boldsymbol{b}=\hat{\boldsymbol{B}} \exp (\mathrm{j} \omega t)$ and $\boldsymbol{c}=\hat{\boldsymbol{C}} \exp (\mathrm{j} \omega t)$, see Eqs. (12)-(14) above. $\boldsymbol{M}$ designates the overall system mass matrix and $\boldsymbol{K}$ the stiffness one, while $\boldsymbol{f}=\hat{\boldsymbol{F}} \exp (\mathrm{j} \omega t)$ is the force vector. The detailed expressions and calculation details of matrices $\boldsymbol{M}, \boldsymbol{K}$ and vector $\boldsymbol{F}$ are exposed in Appendix A. The matrices are first computed analytically for a shell of uniform thickness $h_{\text {uni. }}$. Then, the region where the ABH indentation is to be placed is removed from $\boldsymbol{M}$ and $\boldsymbol{K}$ and substituted with that of the ABH profile plus the attached viscoelastic layer. Their contribution to $\boldsymbol{M}$ and $\boldsymbol{K}$ is evaluated through numerical integration. This approach has been termed the matrix-replacing strategy in previous works (see [21] for details). It allows one to efficiently deal with ABH indentations on structures, like the annular one for cylindrical shells in this paper.

Finally, applying the Euler-Lagrange equations $\partial_{t}\left(\partial_{\dot{q}} \mathscr{L}\right)-\partial_{\boldsymbol{q}} \mathscr{L}=0$ to Eq. (21) results in the system equations of motion, which in the frequency domain read,

$$
\left(-\omega^{2} \boldsymbol{M}+\boldsymbol{K}\right) \hat{\boldsymbol{Q}}=\hat{\boldsymbol{F}} .
$$


The solution to Eq. (22) provides the ABH shell displacement field for a given frequency value. For the examples in this work, the size of the GEM mass and stiffness matrices in Eq. (22) are not very large and a direct solver can be used to solve the linear system. Alternatively, one could resort to iterative solvers like the well-known generalized minimal residual method (GMRES), among others, or simply deal with reduced order models by typically projecting onto a low-order modal basis and dealing with reduced mass and stiffness matrices (see e.g., [51]). The modes can be computed by setting $\hat{\boldsymbol{F}}=\mathbf{0}$ in Eq. (22), which leaves us with a generalized eigenvalue problem,

$$
\left(-\omega^{2} \boldsymbol{M}+\boldsymbol{K}\right) \hat{\boldsymbol{Q}}=\mathbf{0}
$$

with eigenpairs $\left(\omega_{n}, \hat{\boldsymbol{Q}}_{n}\right), \hat{\boldsymbol{Q}}_{n}=\left[\hat{\boldsymbol{A}}_{n}^{\top}, \hat{\boldsymbol{B}}_{n}^{\top}, \hat{\boldsymbol{C}}_{n}^{\top}\right]^{\top}$. The radial components of the structural modal shapes can be recovered through

$$
w_{n}(x, y)=\hat{C}_{n}^{\top} \varphi(x, y)
$$

\subsection{Periodicity conditions in the circumferential direction}

The developments in the previous section would suffice to characterize an open cylindrical shell with a line discontinuity at say, $y=-\pi R$. However, they cannot be used to calculate the response of a standard cylindrical shell unless the basis function vectors $\boldsymbol{\beta}^{k}(k=\psi, \xi, \varphi)$ fulfill continuity between $y=-\pi R$ and $y=\pi R$. Note that in this work we are using Gaussian bases to reconstruct both, the displacement fields in the axial and circumferential directions. As for the latter one could have alternatively chosen a harmonic basis but designs with $\mathrm{ABH}$ embedded in the circumferential direction are also feasible (see e.g., the $\mathrm{ABH}$ circular beams in [50]). The use of a Gaussian basis in both directions facilitates dealing with more complex $\mathrm{ABH}$ designs and latter in this work enables the inclusion of stiffeners, which make the cylindrical shell thickness no longer constant in the circumferential direction, for a fixed axial position.

Continuity is required for the displacement field and its first and second derivatives, as observed from Eqs. (7) and (9). The following conditions must hold,

$$
\begin{gathered}
u(x,-\pi R)=u(x, \pi R), v(x,-\pi R)=v(x, \pi R), w(x,-\pi R)=w(x, \pi R), \\
\frac{\partial u}{\partial y}(x,-\pi R)=\frac{\partial u}{\partial y}(x, \pi R), \frac{\partial v}{\partial y}(x,-\pi R)=\frac{\partial v}{\partial y}(x, \pi R), \frac{\partial w}{\partial y}(x,-\pi R)=\frac{\partial w}{\partial y}(x, \pi R), \\
\frac{\partial^{2} u}{\partial y^{2}}(x,-\pi R)=\frac{\partial^{2} u}{\partial y^{2}}(x, \pi R), \frac{\partial^{2} v}{\partial y^{2}}(x,-\pi R)=\frac{\partial^{2} v}{\partial y^{2}}(x, \pi R), \frac{\partial^{2} w}{\partial y^{2}}(x,-\pi R)=\frac{\partial^{2} w}{\partial y^{2}}(x, \pi R) .
\end{gathered}
$$

To satisfy Eqs. (25)-(27), the shape functions in Eqs. (15)-(17) need to be modified. The detailed procedure to construct a proper set of Gaussian basis functions for the cylindrical shell is explained in Appendix B.

\section{Validation of the Gaussian expansion method}

In this section, we will validate the proposed GEM for ABH cylindrical shells against finite element (FEM) simulations. The geometrical and material properties of the tested cylindrical shell and its annular $\mathrm{ABH}$ indentation are summarized in Table 1. The Gaussian basis has been constructed taking $\delta_{x}=\delta_{y}=3$ in Eq. (19), which results in $q_{x}=[-44,44]$ for Eq. (20) and $q_{y}=[-30,30]$ for its analogous in the circumferential direction. After applying the periodic boundary conditions for the latter, the range of $q_{y}$ gets reduced to $q_{y}=[-27,27]$. The final dimensions of the basis function vectors become $\boldsymbol{\psi}_{4895 \times 1}=\boldsymbol{\xi}_{4895 \times 1}=\boldsymbol{\varphi}_{4895 \times 1}=$ $\boldsymbol{\alpha}_{89 \times 1}^{\varphi} \otimes \boldsymbol{\beta}_{55 \times 1}^{\varphi}$ and those of the mass and stiffness matrices $\boldsymbol{M}_{14685 \times 14685}$ and $\boldsymbol{K}_{14685 \times 14685}$. On the other hand, the reference FEM model has been built using the commercial software package Comsol. A structured mesh of quadrilaterals has been used for the cylindrical shell having a characteristic element size of $2 \mathrm{~cm}$. This 

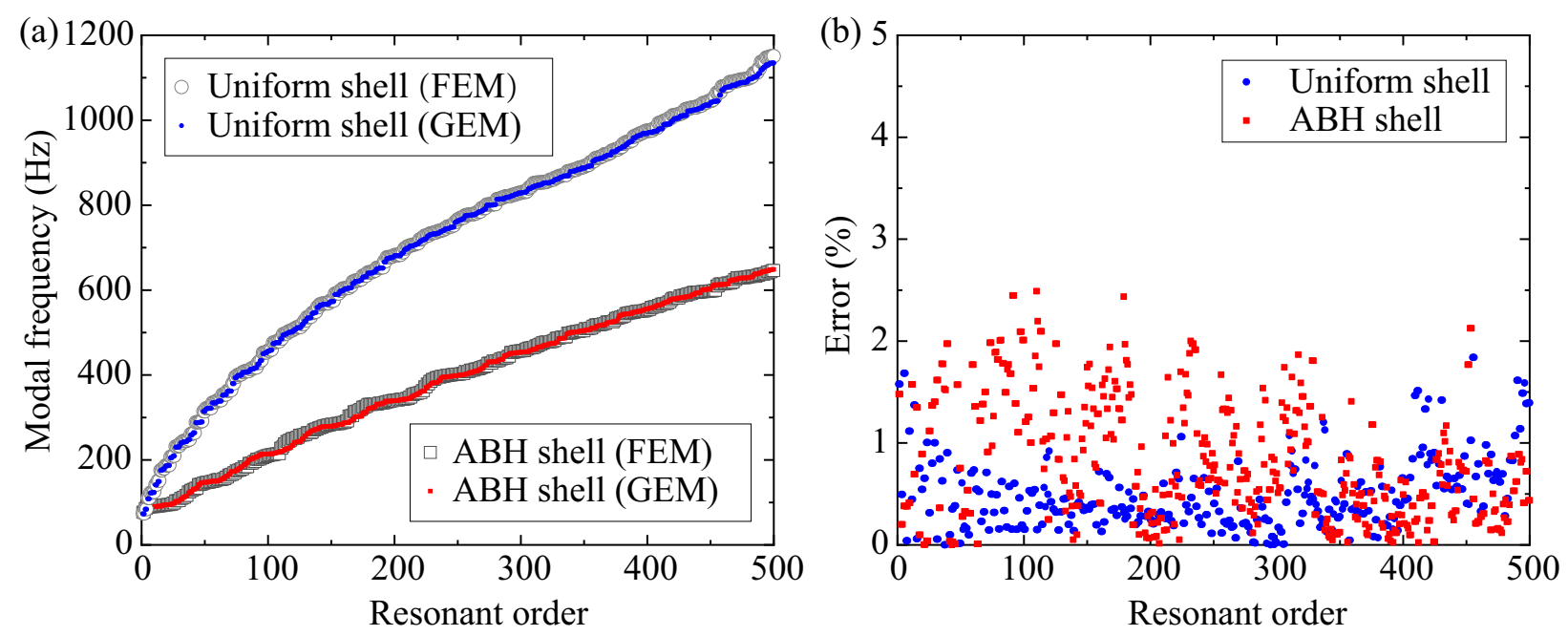

Figure 2: GEM validation against FEM. Comparison of modal frequencies.

has resulted in 79,316 quadratic elements and 317,896 nodes, corresponding to a total of 1,907,376 degrees of freedom. The two ends of the shell have been clamped in the FEM simulations. This has been emulated in the GEM by taking the following values for the line-distributed boundary springs: $k_{1}=k_{2}=1 \times 10^{10} \mathrm{~N} / \mathrm{m}^{2}$ and $p_{1}=p_{2}=1 \times 10^{10} \mathrm{~N}$.

The computational run lasted for $528 \mathrm{~s}$ to recover the first 500 modes via the eigenvalue module in Comsol, while it took only $203 \mathrm{~s}$ with a Matlab implementation of the proposed method (both calculations were performed on a personal computer Intel i5 $2.50 \mathrm{GHz}$ ). As it will be next shown, the GEM thus offers a very good compromise between accuracy and computational cost.

To begin the validation, we have computed the resonant modes for a uniform cylindrical shell and a cylindrical shell with an embedded annular ABH, using both the FEM and GEM approaches. The first 500 modes and their respective eigenfrequencies have been plotted in Fig. 2a. As observed, the values obtained for the uniform and ABH cylindrical shells with the GEM are very close to those of FEM, and it becomes difficult to distinguish any difference between them. For a better inspection, we have plotted the relative errors between both methods in Fig. 2b. It can be seen that all errors are beneath $3 \%$ and the vast majority hardly surpass $2 \%$. The relative errors of the $\mathrm{ABH}$ shell are slightly higher than those of the uniform shell because the same integration scheme has been used for them in the GEM, the geometry of the ABH shell being more demanding.

\begin{tabular}{cc}
\hline Geometry parameters & Material parameters \\
\hline$m=3$ & $\rho=7800 \mathrm{~kg} / \mathrm{m}^{3}$ \\
$a=2.5 \mathrm{~m}$ & $E=210 \mathrm{GPa}$ \\
$R=1 \mathrm{~m}$ & $\eta=0.005$ \\
$h_{\mathrm{uni}}=0.02 \mathrm{~m}$ & $\nu=0.3$ \\
$r_{\mathrm{abh}}=1.25 \mathrm{~m}$ & \\
$\varepsilon=0.0097 \mathrm{~m}^{-2}$ & $\rho_{v}=950 \mathrm{~kg} / \mathrm{m}^{3}$ \\
$h_{c}=0.001 \mathrm{~m}$ & $E_{v}=5 \mathrm{GPa}$ \\
$r_{v}=1.25 \mathrm{~m}$ & $\eta_{v}=0.5$ \\
$h_{v}=0.005 \mathrm{~m}$ & $\nu_{v}=0.3$ \\
\hline
\end{tabular}

Table 1: Geometry and material parameters of the ABH cylindrical shell. $\rho$ : shell density, $\rho_{v}$ : damping layer density, $E$ : shell Young modulus, $E_{v}$ : damping layer Young modulus, $\eta$ : shell loss factor, $\eta_{v}$ : damping layer loss factor, $\nu$ : shell Poisson ratio, $\nu_{v}$ : damping layer Poisson ratio. 


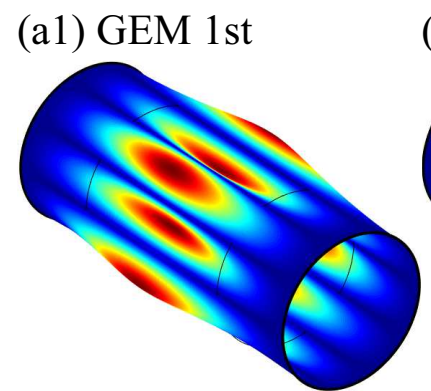

(b1) FEM 1st

(a2) GEM 8th

(a3) GEM 25th

(a4) GEM 40th

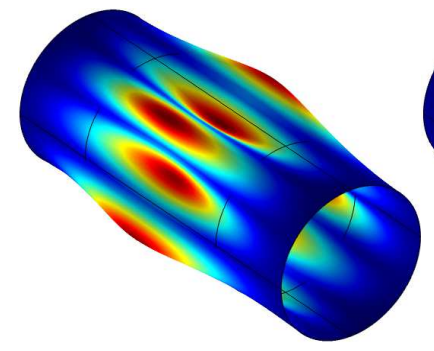

(b2) FEM 8th
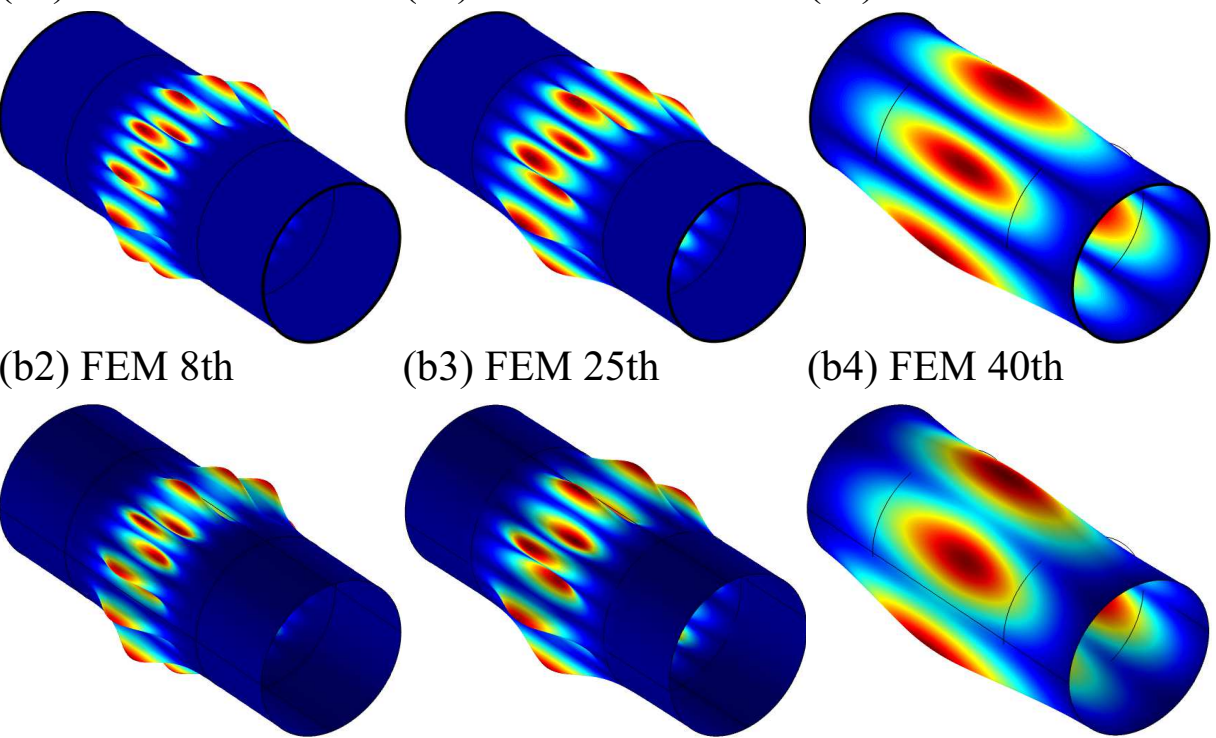

(b3) FEM 25th

(b4) FEM 40th
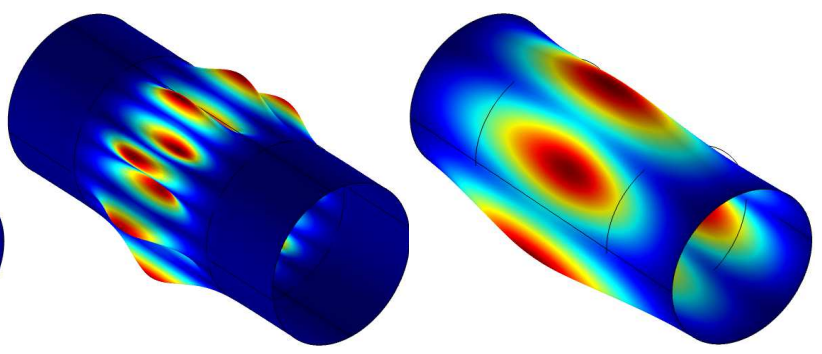

(a5) GEM 50th

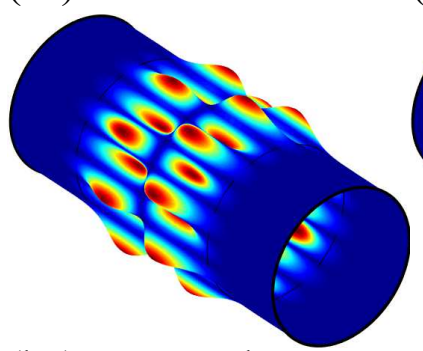

(a6) GEM 70th

(a7) GEM 110th

(a8) GEM 310th

(b5) FEM 50th
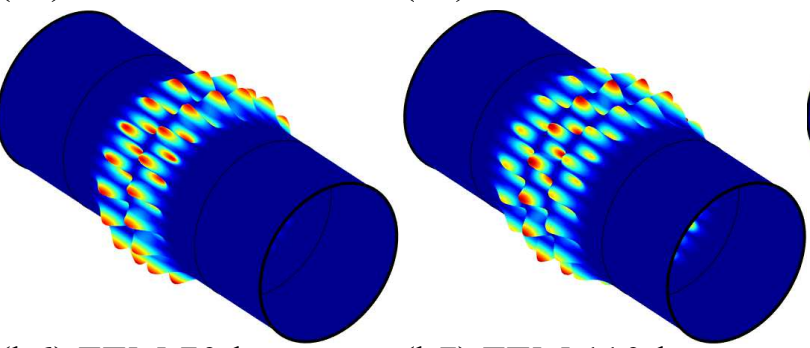

(b7) FEM 110th
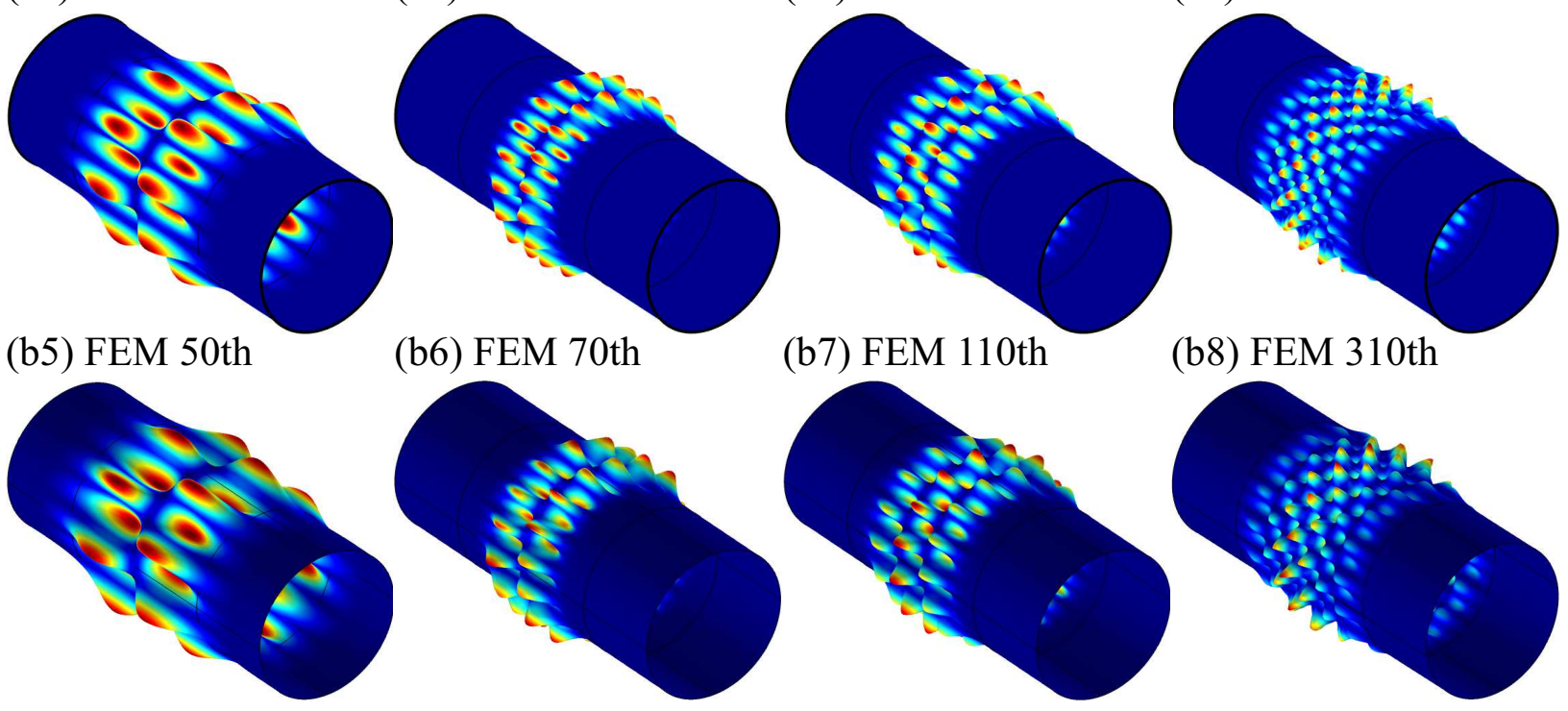

Figure 3: Modal shapes of the ABH cylindrical shell computed with GEM and FEM.

Next, we have computed several modal shapes from Eq. (24) and plotted them in Fig. 3. The first two rows in the figure contain the orders 1, 8, 25 and 40 for the ABH cylindrical shell, respectively computed with the GEM and the FEM. Besides, the third and fourth rows contain the orders 50, 70, 110 and 310, again computed with the GEM and the FEM. It is seen that the ABH indentation strongly amplifies the modal vibration in the middle part of the shell. It can also be observed that the low order modes mainly involve the circumferential direction (first and second rows), while higher order modes also include bending motion in the axial direction. In all cases, the resemblance between the GEM and FEM results is remarkable. On the other hand, and for completeness, in Fig. 4 we also present the modal shapes for the orders 1, 25, 50 and 110 of the uniform shell. As expected, in this case the amplitude is distributed along the whole structure. Again, the GEM results match very well the FEM ones. 

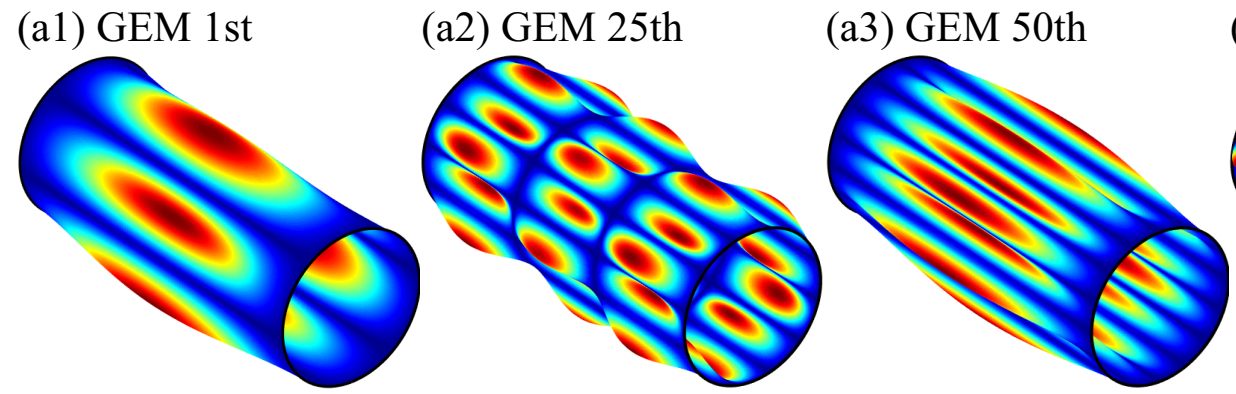

(a4) GEM 110th

(b1) FEM 1st

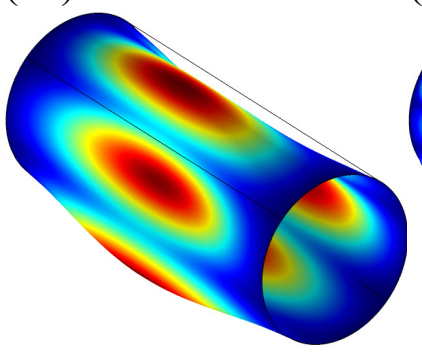

(b2) FEM 25th

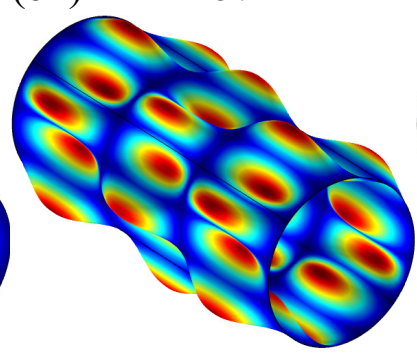

(b3) FEM 50th
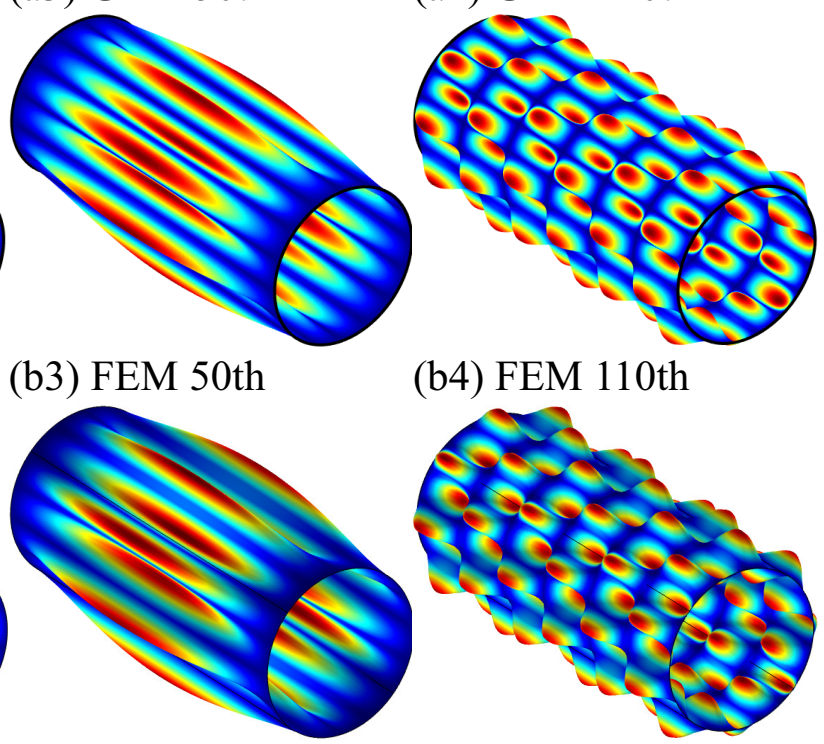

(b4) FEM 110th

Figure 4: Modal shapes of the uniform cylindrical shell computed with GEM and FEM.

Finally, it is worth mentioning that for simplicity in building the FEM models, no damping layers have been considered in this section. As regards validation however, it should be noted that the fully coupled scheme implemented for them in the theoretical GEM model has already been proved accurate by comparison with numerical models and experiments in [18]. All things considered, one may conclude that the GEM offers an accurate and efficient procedure to determine the vibrations of cylindrical shells with embedded annular ABHs.

\section{Simulation results}

\subsection{Annular ABH effects: energy focusing and vibration attenuation}

Let us next analyze the benefits of inserting an annular ABH indentation on a cylindrical shell, in terms of energy concentration and vibration attenuation. To that purpose, we have computed the displacement response of the uniform and $\mathrm{ABH}$ shells up to $1000 \mathrm{~Hz}$, when a point force of amplitude $1 \mathrm{~N} / \mathrm{m}$ is exerted at $\left(x_{f}, y_{f}\right)=(1.875,0) \mathrm{m}$. This point excitation will be assumed in all subsequent simulations unless otherwise specified.

Prior to starting the analysis, however, let us review the role of three threshold frequencies that could have a significant impact on the annular $\mathrm{ABH}$ performance. It is well known that an $\mathrm{ABH}$ starts functioning beyond the so-called diameter cut-on frequency, $f_{r} \approx\left(\pi h_{\mathrm{uni}} / 4 r_{\mathrm{abh}}\right)(E / 3 \rho)^{1 / 2}$, which essentially determines that the wavelength of the incident wave must be smaller than the ABH diameter. Nonetheless, the $\mathrm{ABH}$ profile needs to be also smooth enough for proper impedance matching of the incident wave, so in practice the $\mathrm{ABH}$ effect only becomes fully operative well passed the so-called smoothness cut-on frequency, $f_{\varepsilon 0} \approx$ $(\varepsilon / 2 \pi)(E / 3 \rho)^{1 / 2}$ (typically $3 f_{\varepsilon 0}$ works well, see e.g., [21]). For the problem at hand we are considering a very large $\mathrm{ABH}$ (see Table 1 ) that results in very low diameter and smoothness cut-on frequencies. Actually, we get $f_{r} \approx 37.6 \mathrm{~Hz}$ and $3 f_{\varepsilon 0} \approx 15.9 \mathrm{~Hz}$. Note that in most $\mathrm{ABH}$ designs $3 f_{\varepsilon 0} \gg f_{r}$ but the situation is reversed in this example because of the very big dimensions of the ABH. Taking into account that the analysis of the results reported below in this section will start at $50 \mathrm{~Hz}$, one would a priori expect the $\mathrm{ABH}$ to become effective for the whole considered frequency range. Another important frequency to contemplate for cylindrical shells is the ring frequency, $f_{R} \approx(1 / 2 \pi R)(E / 3 \rho)^{1 / 2}$. It turns out that $f_{R} \approx 865 \mathrm{~Hz}$ in the current case, which means that beyond this frequency the curvature of the cylinder becomes negligible 

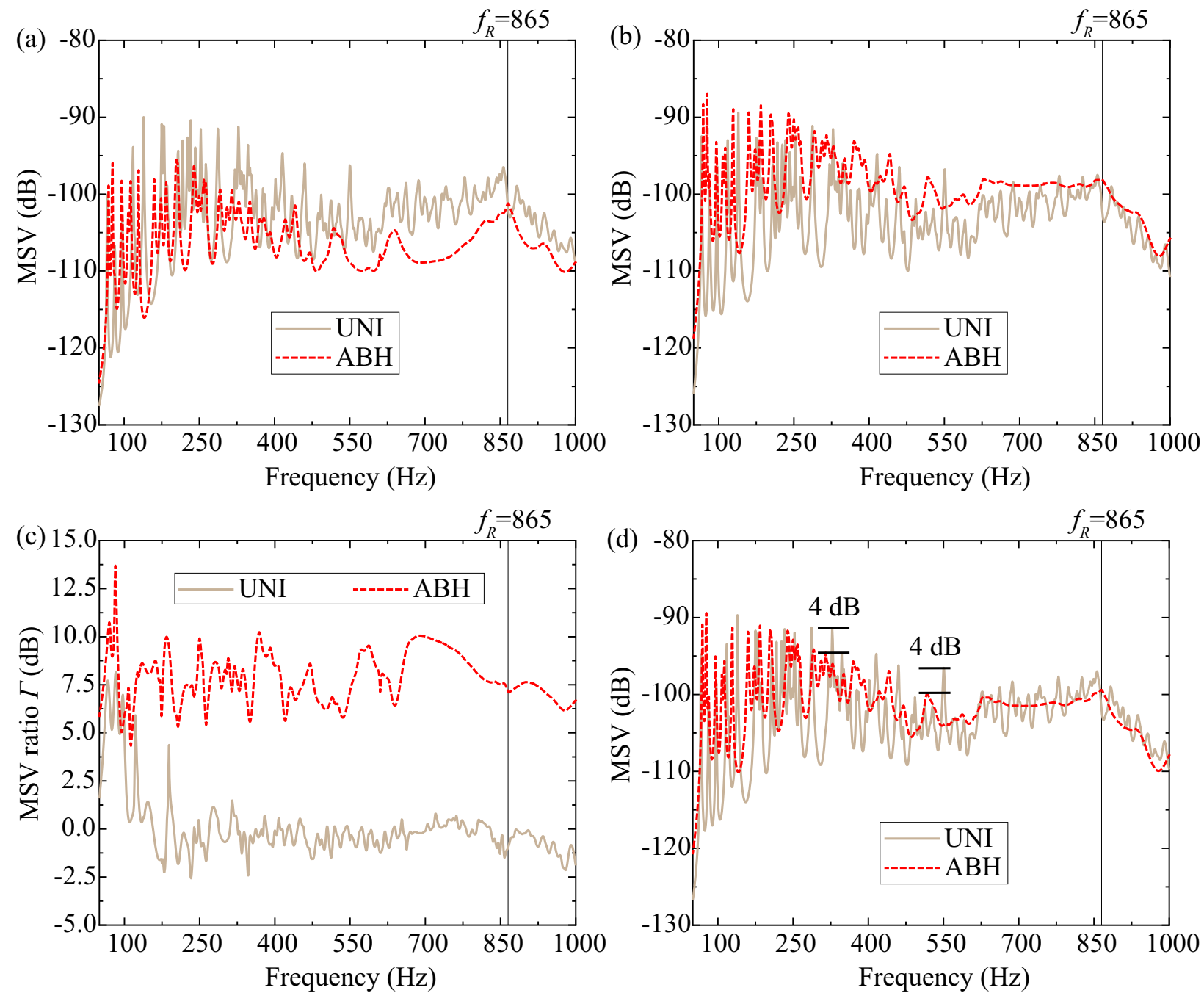

Figure 5: Mean square velocity (MSV) for (a) the uniform portion of the cylindrical shell, (b) the ABH portion and (c) the MSV ratio $\Gamma$ between the $\mathrm{ABH}$ portion and the uniform one, (d) MSV for the overall surface. The legend $U N I$ refers to the uniform cylindrical shell and $A B H$ to the ABH cylindrical shell. The reference velocity is $v_{0}=1 \mathrm{~m} / \mathrm{s}$.

because the shell circumference is much longer than the longitudinal wavelength. Therefore, the cylinder basically behaves as a flat plate for $f>f_{R}$.

Having the above in mind, we can begin the analysis of the simulations. In Fig. 5, we have plotted the surface mean square velocity (MSV) defined as $\left\langle v^{2}\right\rangle=1 / S \int_{S} \omega^{2} w^{2} \mathrm{~d} S$ (and in decibels $\left\langle v^{2}\right\rangle(\mathrm{dB})=$ $10 \log \left(\left\langle v^{2}\right\rangle / v_{0}^{2}\right)$ with $\left.v_{0}=1 \mathrm{~m} / \mathrm{s}\right)$, of different portions $S$ of the ABH cylindrical shell. The latter are compared with the MSV of the correspondig portions $S$ in a uniform cylindrical shell (reference) to illustrate the effects of the annular ABH. As said in section 2, the reference shell has the same amount of damping than the ABH shell for a fair comparison. The MSV for the uniform portion $\left(\left\langle v_{\mathrm{uni}}^{2}\right\rangle\right.$ in the region $(x, y) \in$ $\left.\left[\left(-a,-r_{\mathrm{abh}}\right) \cup\left(r_{\mathrm{abh}}, a\right)\right] \times(-\pi R, \pi R)\right)$ of the $\mathrm{ABH}$ cylindrical shell is compared with that of the reference in Fig. 5a. A noticeable MSV reduction is appreciated as one could expect. Remarkably, however, this takes place for frequencies well beyond $3 f_{\varepsilon 0}$. The reason for that will be explained below. Besides, in Fig. $5 \mathrm{~b}$ we show the comparison between the MSV in the ABH area $\left(\left\langle v_{\mathrm{abh}}^{2}\right\rangle\right.$ in the region $(x, y) \in\left(-r_{\mathrm{abh}}, r_{\mathrm{abh}}\right) \times$ $(-\pi R, \pi R))$ of the shell and the reference. The MSV level for the former is clearly higher, indicating that a large amount of energy concentrates in the $\mathrm{ABH}$ where it can be dissipated by the viscoelastic layer. 


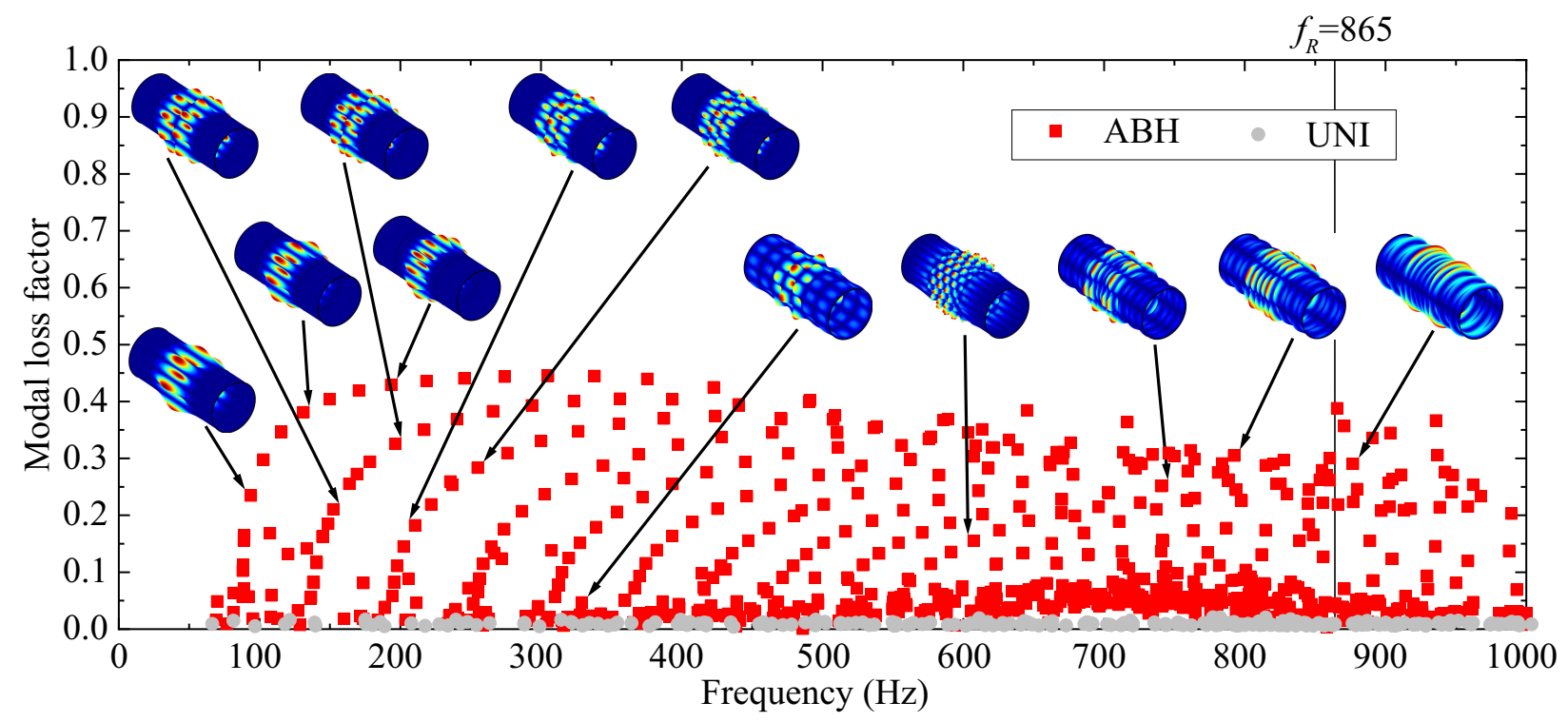

Figure 6: Modal loss factors for both the ABH cylindrical shell and the reference uniform shell. Some modal shapes of the ABH shell are shown in the figure. As observed, circumferential modes dominate for $f \ll f_{R}$ while axial modes appear for frequencies closer to $f_{R}$.

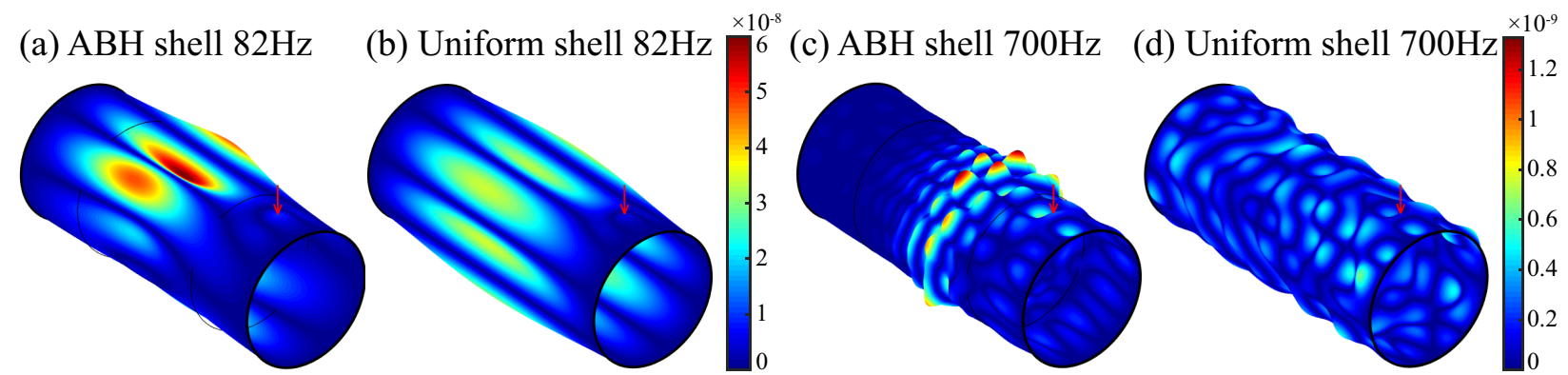

Figure 7: Forced vibration shapes at (a)-(b) $82 \mathrm{~Hz}$ and (c)-(d) $700 \mathrm{~Hz}$. The red arrows indicate the location where the point force is applied.

The MSV ratio defined by $\Gamma=10 \log \left(\left\langle v_{\text {abh }}^{2}\right\rangle /\left\langle v_{\text {uni }}^{2}\right\rangle\right)$ illustrates more clearly this outcome in Fig. 5c. For completion, in Fig. 5d, we plot the overall MSV values, $\left\langle v_{\text {overall }}^{2}\right\rangle$, for the $\mathrm{ABH}$ and reference shells. As observed, some improvement can be appreciated for frequencies above $250 \mathrm{~Hz}$. For instance, a $4 \mathrm{~dB}$ peak reduction is experienced at $\sim 300 \mathrm{~Hz}$ and $\sim 550 \mathrm{~Hz}$ and at higher frequencies the ABH MSV is smaller than that of the uniform shell. Yet, the overall MSV of the ABH shell does not exhibit a striking reduction if compared to that of uniform one. The reason for that is that $\left\langle v_{\text {overall }}^{2}\right\rangle$ is the combination of $\left\langle v_{\text {abh }}^{2}\right\rangle$ and $\left\langle v_{\text {uni }}^{2}\right\rangle$, so the MSV reduction in the uniform part (see Fig. 5a) gets compensated by the augment in the $\mathrm{ABH}$ region (see Fig. 5b). One should be careful though in seeing the overall MSV value as a clear indicator of the good performance of $\mathrm{ABH}$ structures. The main effect of an $\mathrm{ABH}$ is that of extracting vibrational energy from the uniform portion of the shell and transferring it to the central ABH region, where it can be dissipated by the damping layer. This can be exploited in several ways. For instance, having low vibration regions in a structure could be beneficial for coupling to other structural elements. Also, ABHs in plates are known to strongly diminish their sound radiation efficiency as flexural waves become subsonic when propagating inside the ABH region (see e.g., [6]). Such interesting phenomena become masked if one only looks at the overall MSV value of an ABH structure.

As mentioned before, one would predict the $\mathrm{ABH}$ to start functioning beyond $\max \left\{f_{r}, 3 f_{\varepsilon 0}\right\} \approx 50 \mathrm{~Hz}$, 

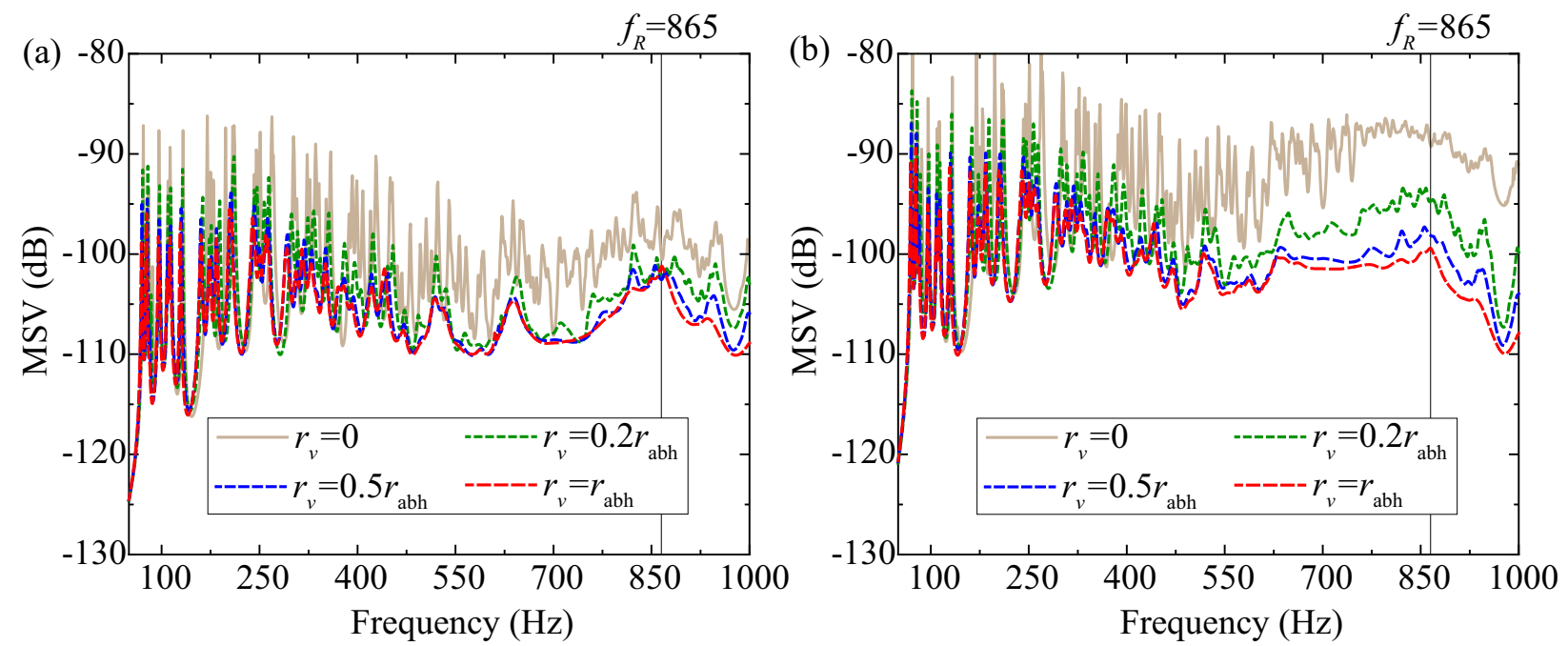

Figure 8: Influence of the size of the damping layer. Mean square velocity (MSV) for (a) the uniform portion of the cylindrical shell and (b) the overall shell surface. The reference velocity is $v_{0}=1 \mathrm{~m} / \mathrm{s}$.

but in the present example we have recognized that this only occurs for $f>250 \mathrm{~Hz}$. The reason for that is that the annular ABH fundamentally attenuates waves in the axial direction while for $f \ll f_{R}$ most modes of the ABH cylindrical shell are circumferential. To prove this assertion, we have calculated the modal loss factors $\eta_{n}=\operatorname{Im}\left(\omega_{n}^{2}\right) / \operatorname{Re}\left(\omega_{n}^{2}\right)$ for both the ABH shell and the reference one. These are presented in Fig. 6, which reveals that the modal loss factors of the $\mathrm{ABH}$ shell are much higher than those of the reference. Most importantly, it is observed in the figure that the higher modal loss factors always correspond to local modes in the $\mathrm{ABH}$ region. For $f \ll f_{R}$, the local modes take place in the circumferential direction and do not trigger the $\mathrm{ABH}$ effect. In addition, the vibration amplitude gets amplified because of the stiffness reduction in the $\mathrm{ABH}$ portion, which results in larger MSV values over the whole surface at low frequencies observed in Fig. 5d. On the other hand, the axial modes appear at high frequencies and get damped by the ABH effect. Further evidence of this behavior can be grasped from the forced vibration shapes of the $\mathrm{ABH}$ and reference shells in Fig. 7. One can readily check how the vibration shapes at $82 \mathrm{~Hz}$ distribute in the circumferential direction (see Fig. 7a) resulting in little energy absorption by the damping layers. As opposed, at a higher frequency like $700 \mathrm{~Hz}$ (see Fig. 7b), bending waves propagate and the annular $\mathrm{ABH}$ increases its efficiency in reducing vibrations (compare the forced shapes of the ABH and uniform shells in the figure). Getting back to Fig. 5, it is interesting to note how in all subfigures the MSV decreases once surpassed the ring frequency $f_{R}=865 \mathrm{~Hz}$, when the cylinder starts behaving like a flat plate.

\subsection{Influence of the size of the viscoelastic damping layer}

It is well-known that the viscous damping layer plays a critical role in the ABH effect by dissipating the energy that concentrates at its center. The influence of the size, shape and thickness of the damping layer has been widely studied in previous works on straight beams [18, 19] and flat plates [21]. In a nutshell, the larger and thicker the damping layer the better, though beyond a certain limit augmenting the damping layer size is no longer beneficial. The latter is confirmed here for the case of annular ABH in cylindrical shells. To this aim, results are presented in Fig. 8 for four different cases characterized by $r_{v} / r_{\text {abh }}=[0,0.2,0.5,1]$. The MSV values in Figs. $8 \mathrm{a}$ and $8 \mathrm{~b}$ indicate that a small amount of damping (e.g. $\left.r_{v}=0.2 r_{\text {abh }}\right)$ makes a big difference with respect to the bare case. Increasing $r_{v} / r_{\text {abh }}$ results in better energy dissipation but values $r_{v} / r_{\text {abh }}>0.5$ induce negligible improvements. For instance, doubling the damping layer material to $r_{v} / r_{\mathrm{abh}}=1$ results in negligible variations. This is consistent with previous conclusions on the influence of the damping layer [21].

To better illustrate the above considerations, in Fig. 9 we present the deflection shapes for the four tested 


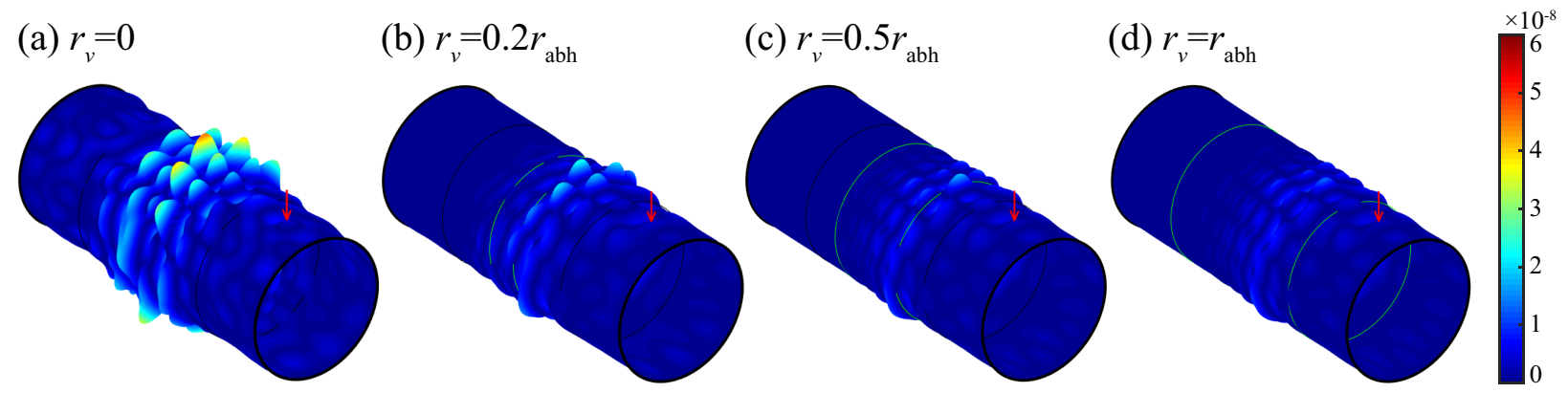

Figure 9: Forced vibration shapes at $750 \mathrm{~Hz}$ for different damping layer sizes. The green circles indicate the boundaries of the damping layers.

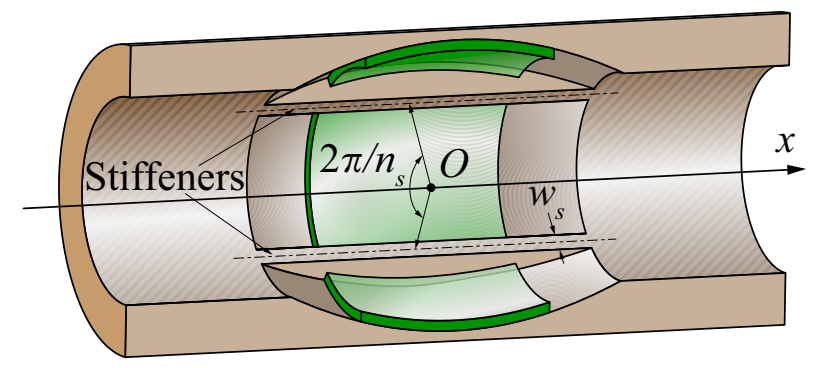

Figure 10: Sketch of the ABH cylindrical shell reinforced with longitudinal stiffeners.
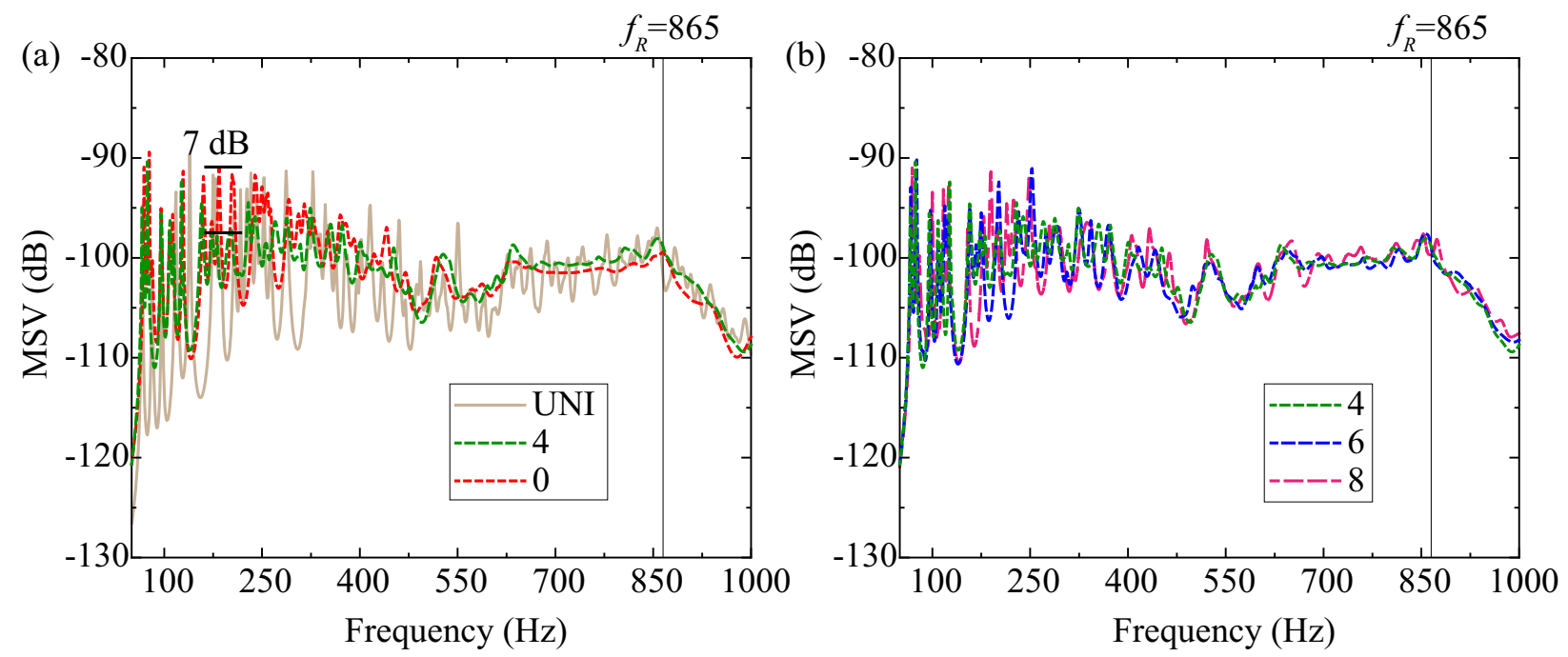

Figure 11: Overall surface MSV comparison among (a) uniform, ABH and 4-stiffener ABH shells (b) 4, 6 and 8-stiffener ABH shells.

damping layer configurations at $750 \mathrm{~Hz}$. The figure confirms the uselessness of incrementing the size of the damping layer beyond $r_{v}=0.5 r_{\text {abh }}$ because dissipation takes place in a much smaller area closer to the ABH center. Therefore, for practical implementations taking $r_{v}=0.5 r_{\text {abh }}$ would suffice.

\subsection{Inclusion of longitudinal stiffeners}

ABHs have the ability of reducing the vibrations of a structure with the remarkable property of diminishing its mass. However, this also poses a disadvantage in terms of structural stiffness. If the thickness at 
(a) ABH 0 stiffener

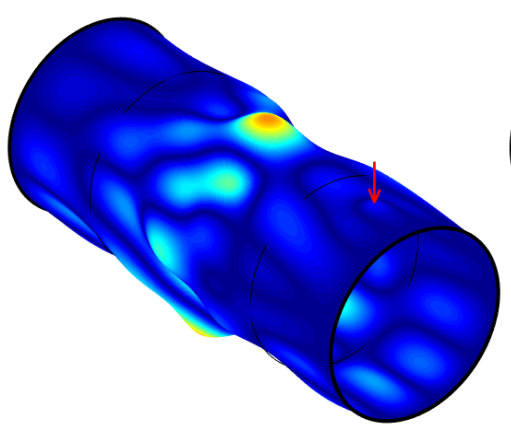

(b) $\mathrm{ABH} 4$ stiffeners

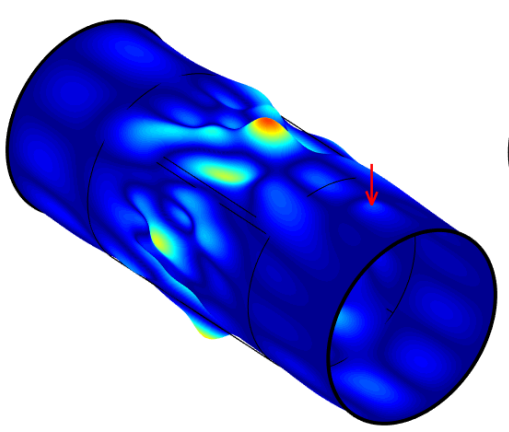

(c) Uniform

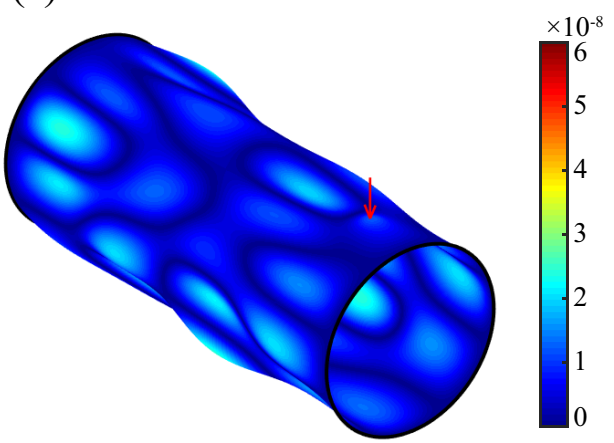

Figure 12: Forced vibration shapes at $250 \mathrm{~Hz}$ for (a) ABH shell without stiffeners, (b) ABH shell with 4 stiffeners and (c) uniform shell.

the $\mathrm{ABH}$ central section is too thin, an ABH cylindrical shell could collapse under excessive external load. To prevent that situation one can insert longitudinal stiffeners in the structure. The inclusion of stiffeners in ABHs was analyzed for the case of annular ABHs in plates in [22] and numerically tested for cylindrical shells in the very recent work [24]. Hereafter, we will use the GEM in combination with the matrix-replacing strategy [21] to characterize the insertion of longitudinal stiffeners having the thickness of the uniform part of the shell, the same length of the $\mathrm{ABH}$ and width $w_{s}$. A 3D sketch of the proposed annular $\mathrm{ABH}$ with stiffeners is presented in Fig. 10.

To begin with, we compare the MSV of a point forced ABH with 4 uniformly distributed stiffeners of width $w_{s}=0.08 \mathrm{~m}$, with that of an $\mathrm{ABH}$ without stiffeners and with the reference uniform shell. The results are plotted in Fig. 11a. It is seen in the figure that the stiffeners help attenuating the vibration below $500 \mathrm{~Hz}$, especially in the frequency range [150,300] Hz. The MSV of the ABH stiffened shell decreases $\sim 7 \mathrm{~dB}$ at some frequencies when compared to that of the non-stiffened ABH shell. This result is logical since the high MSV levels encountered for the ABH shell at that frequency range were attributed to weak structural rigidity and to the excitation of circumferential modes. Both aspects are compensated with the inclusion of the stiffeners. This is a significant result because a priori one would not automatically infer that the stiffeners do not only reinforce the structure but also help diminishing low frequency vibrations. In fact, this behavior was recently reported in [24] though no physical explanation was given for it. Nonetheless, beyond $600 \mathrm{~Hz}$, where the ABH is fully operative, the presence of the ABHs is slightly detrimental. This is recognized in Fig. $11 \mathrm{~b}$ as the number of stiffeners increases. The ABH dissipation area diminishes and vibrations can be transmitted through them.

For completion, in Fig. 12, we have plotted the forced vibration shapes at $250 \mathrm{~Hz}$ of the stiffened $\mathrm{ABH}$, non stiffened $\mathrm{ABH}$ and reference shell analyzed in Fig. 11a. At this frequency the overall MSV value of the stiffened $\mathrm{ABH}$ is $\sim 7-8 \mathrm{~dB}$ lower than that of the non stiffened $\mathrm{ABH}$.

\section{Conclusions}

In this paper, we have shown how the Gaussian expansion method (GEM) can be applied to a cylindrical shell with an embedded annular acoustic black hole (ABH). The GEM uses Gaussian shape functions in the framework of the Rayleigh-Ritz method and has the feature of accurately reproducing the vibration field in the thinner portion of an $\mathrm{ABH}$, at a very reasonable computational cost. The key for the GEM to work properly for cylindrical shells is that of building a basis of Gaussian functions that satisfy the periodic boundary conditions in the circumferential direction. This can be done quite straightforwardly within the GEM.

After validating the proposed GEM approach with a finite element model, we have applied it to characterize the vibration attenuation and energy concentration of the annular ABH. Whereas the cut-on frequency at which the $\mathrm{ABH}$ effect is fully operational in flat plates is roughly dictated by three times the smoothness 
frequency, this is not the case for the annular $\mathrm{ABH}$. The latter is essentially designed to reduce waves propagating in the axial direction of the cylindrical shell. However, the low frequency range of the ABH mean square velocity spectrum in the analyzed example is governed by waves in the circumferential direction. The ABH is useless for them and it is not until bending modes get excited that the annular ABH starts functioning well. This is the reason why including some stiffeners to strengthen the shell has turned out to be beneficial at low frequencies: the structural rigidity increases and exciting circumferential modes becomes more difficult. For higher frequencies, though, the stiffeners worsen the ABH effect. For the example at hand, it has been found that inserting a total of four stiffeners provides a good balance between the performance at lower and higher frequencies. On the other hand, the amount of damping needed for proper energy dissipation at the ABH center has also been investigated. Here, the same as for ABHs on flat surfaces can be concluded. Covering the central half of the $\mathrm{ABH}$ area totally suffices for dissipation purposes, the inclusion of additional viscous material being useless.

The current work contributes to showing the potential of $\mathrm{ABH}$ designs and methods for curved structural elements commonly found in many areas of engineering. Future optimized ABH profiles that contemplate, for instance, the coupling among flexural, extensional and shear waves due to curvature, could be worth exploring.

\section{Acknowledgements}

This work has been completed while the first author was performing a two-year PhD stay at La Salle, Universitat Ramon Llull, funded by the National Natural Science Foundation of China under Grant (51875061) and the China Scholarship Council (CSC No.201806050075). The authors gratefully acknowledge this support as well as the in-kind assistance from La Salle, Universitat Ramon Llull, and the Chongqing University to make this collaboration possible. In addition, the authors acknowledge Hang Wu, Ziwei Zhang, Xuqiang Qiao and Yinghong Yu for their help with some of the computations.

\section{Appendix A: Mass matrix, stiffness matrix and force vector}

The mass matrix $\boldsymbol{M}$ is given by

$$
\boldsymbol{M}=\left[\begin{array}{ccc}
\boldsymbol{M}^{u u} & \mathbf{0} & \mathbf{0} \\
& \boldsymbol{M}^{v v} & \mathbf{0} \\
\text { sym. } & & \boldsymbol{M}^{w w}
\end{array}\right],
$$

where

$$
\begin{aligned}
\boldsymbol{M}^{u u} & =\rho \int_{-\pi R}^{\pi R} \int_{-a}^{a} h(x, y) \boldsymbol{\psi}(x, y) \boldsymbol{\psi}^{\top}(x, y) \mathrm{d} x \mathrm{~d} y=\rho \int_{-\pi R}^{\pi R} \int_{-a}^{a} h(x, y)\left(\boldsymbol{\alpha}^{\psi} \boldsymbol{\alpha}^{\psi^{\top}}\right) \otimes\left(\boldsymbol{\beta}^{\psi} \boldsymbol{\beta}^{\psi^{\top}}\right) \mathrm{d} x \mathrm{~d} y, \\
\boldsymbol{M}^{v v} & =\rho \int_{-\pi R}^{\pi R} \int_{-a}^{a} h(x, y) \boldsymbol{\xi}(x, y) \boldsymbol{\xi}^{\top}(x, y) \mathrm{d} x \mathrm{~d} y=\rho \int_{-\pi R}^{\pi R} \int_{-a}^{a} h(x, y)\left(\boldsymbol{\alpha}^{\xi} \boldsymbol{\alpha}^{\xi^{\top}}\right) \otimes\left(\boldsymbol{\beta}^{\xi} \boldsymbol{\beta}^{\xi^{\top}}\right) \mathrm{d} x \mathrm{~d} y, \\
\boldsymbol{M}^{w w} & =\rho \int_{-\pi R}^{2 \pi R} \int_{-a}^{a} h(x, y) \boldsymbol{\varphi}(x, y) \boldsymbol{\varphi}^{\top}(x, y) \mathrm{d} x \mathrm{~d} y=\rho \int_{-\pi R}^{\pi R} \int_{-a}^{a} h(x, y)\left(\boldsymbol{\alpha}^{\varphi} \boldsymbol{\alpha}^{\varphi}\right) \otimes\left(\boldsymbol{\beta}^{\varphi} \boldsymbol{\beta}^{\varphi}\right) \mathrm{d} x \mathrm{~d} y .
\end{aligned}
$$

The components of $\boldsymbol{\alpha}^{k}(x), k=\psi, \xi, \varphi$, are obtained from Eq. (18) and analogous expressions can be derived for $\boldsymbol{\beta}^{k}(y), k=\psi, \xi, \varphi$. Explicit expressions for them satisfying the periodicity in the circumferential direction are derived in Appendix B.

On the other hand, the stiffness matrix $\boldsymbol{K}$ is composed of four parts, namely

$$
\boldsymbol{K}=\boldsymbol{K}_{b e}+\boldsymbol{K}_{e x}+\boldsymbol{K}_{c o}+\boldsymbol{K}_{e d}
$$


Here, $\boldsymbol{K}_{b e}, \boldsymbol{K}_{e x}, \boldsymbol{K}_{c o}$ and $\boldsymbol{K}_{e d}$ respectively represent the contributions to the stiffness matrix caused by the bending motion, the extensional motion, the coupling motion and the boundary conditions at the shell edge.

The bending stiffness matrix is

$$
\boldsymbol{K}_{b e}=\left[\begin{array}{ccc}
\mathbf{0} & \mathbf{0} & \mathbf{0} \\
& \mathbf{0} & \mathbf{0} \\
\text { sym. } & & \boldsymbol{K}_{b e}^{w w}
\end{array}\right],
$$

where

$$
\begin{aligned}
\boldsymbol{K}_{b e}^{w w}= & \int_{-\pi R}^{\pi R} \int_{-a}^{a} D(x, y)\left[\left(\boldsymbol{\alpha}^{\varphi} \boldsymbol{\alpha}^{\varphi \top}\right) \otimes\left(\frac{\mathrm{d}^{2} \boldsymbol{\beta}^{\varphi}}{\mathrm{d} y^{2}} \frac{\mathrm{d}^{2} \boldsymbol{\beta}^{\varphi \top}}{\mathrm{d} y^{2}}\right)+\left(\frac{\mathrm{d}^{2} \boldsymbol{\alpha}^{\varphi}}{\mathrm{d} x^{2}} \frac{\mathrm{d}^{2} \boldsymbol{\alpha}^{\varphi}}{\mathrm{d} x^{2}}\right) \otimes\left(\boldsymbol{\beta}^{\varphi} \boldsymbol{\beta}^{\varphi \top}\right)\right. \\
& +\nu\left(\frac{\mathrm{d}^{2} \boldsymbol{\alpha}^{\varphi}}{\mathrm{d} x^{2}} \boldsymbol{\alpha}^{\varphi \top}\right) \otimes\left(\boldsymbol{\beta}^{\varphi^{\top}} \frac{\mathrm{d}^{2} \boldsymbol{\beta}^{\varphi}}{\mathrm{d} y^{2}}\right)+\nu\left(\boldsymbol{\alpha}^{\varphi^{\top}} \frac{\mathrm{d}^{2} \boldsymbol{\alpha}^{\varphi \top}}{\mathrm{d} x^{2}}\right) \otimes\left(\frac{\mathrm{d}^{2} \boldsymbol{\beta}^{\varphi}}{\mathrm{d} y^{2}} \boldsymbol{\beta}^{\varphi \top}\right) \\
& \left.+2(1-\nu)\left(\frac{\mathrm{d} \boldsymbol{\alpha}^{\varphi}}{\mathrm{d} x} \frac{\mathrm{d} \boldsymbol{\alpha}^{\varphi}}{\mathrm{d} x}\right) \otimes\left(\frac{\mathrm{d} \boldsymbol{\beta}^{\varphi}}{\mathrm{d} y} \frac{\mathrm{d} \boldsymbol{\beta}^{\varphi}}{\mathrm{d} y}\right)\right] \mathrm{d} x \mathrm{~d} y .
\end{aligned}
$$

Besides, the extensional stiffness matrix has the expression

$$
\boldsymbol{K}_{e x}=\left[\begin{array}{ccc}
\boldsymbol{K}_{e x}^{u u} & \boldsymbol{K}_{e x}^{u v} & \boldsymbol{K}_{e x}^{u w} \\
& \boldsymbol{K}_{e x}^{v v} & \boldsymbol{K}_{e x}^{v w} \\
\text { sym. } & & \boldsymbol{K}_{e x}^{w w}
\end{array}\right]
$$

with

$$
\begin{gathered}
\boldsymbol{K}_{e x}^{u u}=\int_{-\pi R}^{\pi R} \int_{-a}^{a} G(x, y)\left[\frac{1-\nu}{2}\left(\boldsymbol{\alpha}^{\psi} \boldsymbol{\alpha}^{\psi^{\top}}\right) \otimes\left(\frac{\mathrm{d} \boldsymbol{\beta}^{\psi}}{\mathrm{d} y} \frac{\mathrm{d} \boldsymbol{\beta}^{\psi^{\top}}}{\mathrm{d} y}\right)+\left(\frac{\mathrm{d} \boldsymbol{\alpha}^{\psi}}{\mathrm{d} x} \frac{\mathrm{d} \boldsymbol{\alpha}^{\top}}{\mathrm{d} x}\right) \otimes\left(\boldsymbol{\beta}^{\psi} \boldsymbol{\beta}^{\psi^{\top}}\right)\right] \mathrm{d} x \mathrm{~d} y, \quad(\mathrm{~A} \theta) \\
\boldsymbol{K}_{e x}^{u v}=\int_{-\pi R}^{\pi R} \int_{-a}^{a} G(x, y)\left[\nu\left(\frac{\mathrm{d} \boldsymbol{\alpha}^{\psi}}{\mathrm{d} x} \boldsymbol{\alpha}^{\xi^{\top}}\right) \otimes\left(\boldsymbol{\beta}^{\psi} \frac{\mathrm{d} \boldsymbol{\beta}^{\xi^{\top}}}{\mathrm{d} y}\right)+\frac{1-\nu}{2}\left(\boldsymbol{\alpha}^{\psi} \frac{\mathrm{d} \boldsymbol{\alpha}^{\xi}}{\mathrm{d} x}\right) \otimes\left(\frac{\mathrm{d} \boldsymbol{\beta}^{\psi}}{\mathrm{d} y} \boldsymbol{\beta}^{\xi^{\top}}\right)\right] \mathrm{d} x \mathrm{~d} y, \\
\boldsymbol{K}_{e x}^{u w}=\int_{-\pi R}^{\pi R} \int_{-a}^{a} G(x, y) \frac{\nu}{R}\left(\frac{\mathrm{d} \boldsymbol{\alpha}^{\psi}}{\mathrm{d} x} \boldsymbol{\alpha}^{\varphi \top}\right) \otimes\left(\boldsymbol{\beta}^{\psi} \boldsymbol{\beta}^{\varphi}\right) \mathrm{d} x \mathrm{~d} y, \\
\int_{-\pi R}^{\pi R} \int_{-a}^{a} G(x, y)\left[\left(\boldsymbol{\alpha}^{\xi} \boldsymbol{\alpha}^{\xi}\right) \otimes\left(\frac{\mathrm{d} \boldsymbol{\beta}^{\xi}}{\mathrm{d} y} \frac{\mathrm{d} \boldsymbol{\beta}^{\xi}}{\mathrm{d} y}\right)+\frac{1-\nu}{2}\left(\frac{\mathrm{d} \boldsymbol{\alpha}^{\xi}}{\mathrm{d} x} \frac{\mathrm{d} \boldsymbol{\alpha}^{\xi}}{\mathrm{d} x}\right) \otimes\left(\boldsymbol{\beta}^{\xi} \boldsymbol{\beta}^{\xi}\right)\right] \mathrm{d} x \mathrm{~d} y, \quad(\mathrm{~A} \\
\boldsymbol{K}_{e x}^{v w}=\int_{-\pi R}^{\pi R} \int_{-a}^{a} G(x, y) \frac{1}{R}\left(\boldsymbol{\alpha}^{\xi} \boldsymbol{\alpha}^{\varphi}\right) \otimes\left(\frac{\mathrm{d} \boldsymbol{\beta}^{\xi}}{\mathrm{d} y} \boldsymbol{\beta}^{\varphi \top}\right) \mathrm{d} x \mathrm{~d} y, \\
\boldsymbol{K}_{e x}^{w w}=\int_{-\pi R}^{\pi R} \int_{-a}^{a} G(x, y) \frac{1}{R^{2}}\left(\boldsymbol{\alpha}^{\varphi} \boldsymbol{\alpha}^{\varphi}\right) \otimes\left(\boldsymbol{\beta}^{\varphi} \boldsymbol{\beta}^{\varphi}\right) \mathrm{d} x \mathrm{~d} y .
\end{gathered}
$$

The coupling stiffness matrix is built as

$$
\boldsymbol{K}_{c o}=\left[\begin{array}{ccc}
\mathbf{0} & \mathbf{0} & \mathbf{0} \\
& \boldsymbol{K}_{c o}^{v v} & \boldsymbol{K}_{c o}^{v w} \\
\text { sym. } & & \mathbf{0}
\end{array}\right]
$$


in which

$$
\boldsymbol{K}_{c o}^{v v}=\int_{-\pi R}^{\pi R} \int_{-a}^{a} D(x, y) \frac{1}{R^{2}}\left[\left(\boldsymbol{\alpha}^{\xi} \boldsymbol{\alpha}^{\xi^{\top}}\right) \otimes\left(\frac{\mathrm{d} \boldsymbol{\beta}^{\xi}}{\mathrm{d} y} \frac{\mathrm{d} \boldsymbol{\beta}^{\xi^{\top}}}{\mathrm{d} y}\right)+2(1-\nu)\left(\frac{\mathrm{d} \boldsymbol{\alpha}^{\xi}}{\mathrm{d} x} \frac{\mathrm{d} \boldsymbol{\alpha}^{\xi^{\top}}}{\mathrm{d} x}\right) \otimes\left(\boldsymbol{\beta}^{\xi} \boldsymbol{\beta}^{\xi^{\top}}\right)\right] \mathrm{d} x \mathrm{~d} y,
$$

and

$$
\begin{aligned}
\boldsymbol{K}_{c o}^{v w}= & -\int_{-\pi R}^{\pi R} \int_{-a}^{a} D(x, y) \frac{1}{R}\left[\left(\boldsymbol{\alpha}^{\xi} \boldsymbol{\alpha}^{\varphi^{\top}}\right) \otimes\left(\frac{\mathrm{d} \boldsymbol{\beta}^{\xi}}{\mathrm{d} y} \frac{\mathrm{d}^{2} \boldsymbol{\beta}^{\varphi \top}}{\mathrm{d} y^{2}}\right)+\nu\left(\boldsymbol{\alpha}^{\xi} \frac{\mathrm{d}^{2} \boldsymbol{\alpha}^{\varphi \top}}{\mathrm{d} x^{2}}\right) \otimes\left(\frac{\mathrm{d} \boldsymbol{\beta}^{\xi}}{\mathrm{d} y} \boldsymbol{\beta}^{\varphi \top}\right)\right. \\
& \left.+2(1-\nu)\left(\frac{\mathrm{d} \boldsymbol{\alpha}^{\xi}}{\mathrm{d} x} \frac{\mathrm{d} \boldsymbol{\alpha}^{\varphi \top}}{\mathrm{d} x}\right) \otimes\left(\boldsymbol{\beta}^{\xi} \frac{\mathrm{d} \boldsymbol{\beta}^{\varphi \top}}{\mathrm{d} y}\right)\right] \mathrm{d} x \mathrm{~d} y .
\end{aligned}
$$

As regards the contribution of the springs at the shell edge to the overall stiffness matrix, this becomes

$$
\boldsymbol{K}_{e d}=\left[\begin{array}{ccc}
\boldsymbol{K}_{e d}^{u u} & \mathbf{0} & \mathbf{0} \\
& \boldsymbol{K}_{e d}^{v v} & \mathbf{0} \\
\text { sym. } & & \boldsymbol{K}_{e d}^{w w}
\end{array}\right]
$$

where

$$
\begin{gathered}
\boldsymbol{K}_{e d}^{u u}=k_{1}^{u} \int_{-\pi R}^{\pi R}\left[\boldsymbol{\alpha}^{\psi}(-a) \boldsymbol{\alpha}^{\psi}(-a)^{\top}\right] \otimes\left(\boldsymbol{\beta}^{\psi} \boldsymbol{\beta}^{\psi^{\top}}\right) \mathrm{d} y+k_{2}^{u} \int_{-\pi R}^{\pi R}\left[\boldsymbol{\alpha}^{\psi}(a) \boldsymbol{\alpha}^{\psi}(a)^{\top}\right] \otimes\left(\boldsymbol{\beta}^{\psi} \boldsymbol{\beta}^{\psi^{\top}}\right) \mathrm{d} y, \quad(\mathrm{~A} 19) \\
\boldsymbol{K}_{e d}^{u u}=k_{1}^{v} \int_{-\pi R}^{\pi R}\left[\boldsymbol{\alpha}^{\xi}(-a) \boldsymbol{\alpha}^{\xi}(-a)^{\top}\right] \otimes\left(\boldsymbol{\beta}^{\xi} \boldsymbol{\beta}^{\xi^{\top}}\right) \mathrm{d} y+k_{2}^{v} \int_{-\pi R}^{\pi R}\left[\boldsymbol{\alpha}^{\xi}(a) \boldsymbol{\alpha}^{\xi}(a)^{\top}\right] \otimes\left(\boldsymbol{\beta}^{\xi} \boldsymbol{\beta}^{\xi^{\top}}\right) \mathrm{d} y, \quad(\mathrm{~A} 20) \\
\boldsymbol{K}_{e d}^{w w}=k_{1}^{w} \int_{-\pi R}^{\pi R}\left[\boldsymbol{\alpha}^{\varphi}(-a) \boldsymbol{\alpha}^{\varphi}(-a)^{\top}\right] \otimes\left(\boldsymbol{\beta}^{\varphi} \boldsymbol{\beta}^{\varphi^{\top}}\right) \mathrm{d} y+k_{2}^{w} \int_{-\pi R}^{\pi R}\left[\boldsymbol{\alpha}^{\varphi}(a) \boldsymbol{\alpha}^{\varphi}(a)^{\top}\right] \otimes\left(\boldsymbol{\beta}^{\varphi} \boldsymbol{\beta}^{\varphi^{\top}}\right) \mathrm{d} y \\
+p_{1}^{w} \int_{-\pi R}^{\pi R}\left[\frac{\mathrm{d} \boldsymbol{\alpha}^{\varphi}}{\mathrm{d} x}(-a) \frac{\mathrm{d} \boldsymbol{\alpha}^{\varphi \top}}{\mathrm{d} x}(-a)\right] \otimes\left(\boldsymbol{\beta}^{\varphi} \boldsymbol{\beta}^{\varphi \top}\right) \mathrm{d} y+p_{2}^{w} \int_{-\pi R}^{\pi R}\left[\frac{\mathrm{d} \boldsymbol{\alpha}^{\varphi}}{\mathrm{d} x}(a) \frac{\mathrm{d} \boldsymbol{\alpha}^{\varphi \top}}{\mathrm{d} x}(a)\right] \otimes\left(\boldsymbol{\beta}^{\varphi} \boldsymbol{\beta}^{\varphi}\right) \mathrm{d} y .
\end{gathered}
$$

Finally, the force vector $\hat{\boldsymbol{F}}$ has the expression

$$
\hat{\boldsymbol{F}}=\left[\mathbf{0}^{\top}, \mathbf{0}^{\top}, \hat{\boldsymbol{F}}^{w \top}\right]^{\top} .
$$

where

$$
\hat{\boldsymbol{F}}^{w}=\boldsymbol{\varphi}\left(x_{f}, y_{f}\right)=\boldsymbol{\alpha}^{\varphi}\left(x_{f}\right) \otimes \boldsymbol{\beta}^{\varphi}\left(y_{f}\right) .
$$

All matrices in the above expressions have been computed by means of the matrix-replacing strategy in [21] (see section 2.3.2 in that paper). The integrals for the uniform shell have been carried out analytically while a numerical 2D composite Gaussian integration scheme has been used for the ABH shells. The mathematical software MATLAB has been employed to perform all computations.

\section{Appendix B: Gaussian basis functions satisfying circumferential periodic conditions}

As quoted in the main text, the same Gaussian basis functions can be used for the three displacement variables, i.e. $\boldsymbol{\alpha}^{\psi}=\boldsymbol{\alpha}^{\xi}=\boldsymbol{\alpha}^{\varphi}$ and $\boldsymbol{\beta}^{\psi}=\boldsymbol{\beta}^{\xi}=\boldsymbol{\beta}^{\varphi}$. Therefore, in what follows it will suffice to present how 
to make the basis functions satisfy the periodic conditions in the $y$ direction for the radial displacement $w$, the procedure being analogous for $u$ and $v$.

In the case of a cylindrical shell with a line aperture (i.e. disconnected at $y=-\pi R$ ), the radial displacement $w(x, y, t)$ can be decomposed in terms of Gaussian basis functions, as described by Eqs. (14), (17) and (18),

$$
w(x, y, t)=\boldsymbol{c}(t)^{\top} \boldsymbol{\varphi}(x, y)=\sum_{i=1}^{n} \sum_{j=1}^{m} \alpha_{i}^{\varphi}(x) \beta_{j}^{\varphi}(y) c_{i j}(t),
$$

where $c_{i j}(t)=c_{i}^{x}(t) c_{j}^{y}(t)$ stand for the unknown time dependent coefficients (superscripts $x$ and $y$ respectively designate the coefficients multiplying $\alpha_{i}^{\varphi}(x)$ and $\left.\beta_{j}^{\varphi}(y)\right)$. $n$ and $m$ are the number of basis functions in the $x$ and $y$ directions.

Imposing the condition $w(x,-\pi R)=w(x, \pi R)$ of Eq. (25) using the factorization Eq. (B1) results in $\sum_{j=1}^{m} \beta_{i}^{\varphi}(\pi R) c_{j}^{y}=\sum_{j=1}^{m} \beta_{j}^{\varphi}(-\pi R) c_{j}^{y}$, from which follows

$$
\sum_{j=1}^{m}\left[\beta_{j}^{\varphi}(\pi R)-\beta_{j}^{\varphi}(-\pi R)\right] c_{j}^{y}=0 .
$$

Expanding the $m$-th term in Eq. (B2) as a combination of the remaining $m-1$ ones provides

$$
\sum_{j=1}^{m-1}\left[\beta_{j}^{\varphi}(\pi R)-\beta_{j}^{\varphi}(-\pi R)\right] c_{j}^{y}=-\left[\beta_{m}^{\varphi}(\pi R)-\beta_{m}^{\varphi}(-\pi R)\right] c_{m}^{y},
$$

which allows one to compute the $m$-th coefficient, $c_{m}^{y}$, as

$$
c_{m}^{y}=-\sum_{j=1}^{m-1} \frac{\beta_{j}^{\varphi}(\pi R)-\beta_{j}^{\varphi}(-\pi R)}{\beta_{m}^{\varphi}(\pi R)-\beta_{m}^{\varphi}(-\pi R)} c_{j}^{y} .
$$

If we next substitute $c_{m}^{y}$ into Eq. (B1), it turns out that $\sum_{j=1}^{m} \beta_{j}^{\varphi} c_{j}^{y}=\sum_{j=1}^{m-1} \beta_{j}^{\varphi} c_{j}^{y}+\beta_{m}^{\varphi} c_{m}^{y}$. We can then define new basis functions $\beta_{j}^{\varphi 1}$ satisfying $w(x,-\pi R)=w(x, \pi R)$ as follows

$$
\beta_{j}^{\varphi 1}=\beta_{j}^{\varphi}-C_{j}^{1} \beta_{m}^{\varphi}(j=1,2,3, \ldots, m-1),
$$

where the superscript 1 of $\beta_{j}^{\varphi 1}$ indicates the number of periodic conditions being considered (in this case that of Eq. (25)). The rule also applies to subsequent formulas. In Eq. (B5), the coefficient $C_{j}^{1}$ is given by $C_{j}^{1}=\left[\beta_{j}^{\varphi}(\pi R)-\beta_{j}^{\varphi}(-\pi R)\right] /\left[\beta_{m}^{\varphi}(\pi R)-\beta_{m}^{\varphi}(-\pi R)\right]$.

We can proceed in analogous manner to impose the additional periodicity conditions of Section 3.2. Applying $\partial_{y} w(x,-\pi R)=\partial_{y} w(x, \pi R)$ of Eq. (26) to the new basis $\beta_{j}^{\varphi 1}$, we can get $c_{m-1}^{y}$ from the remaining $m-2$ coefficients,

$$
c_{m-1}^{y}=-\sum_{j=1}^{m-2} \frac{\partial_{y} \beta_{j}^{\varphi 1}(\pi R)-\partial_{y} \beta_{j}^{\varphi 1}(-\pi R)}{\partial_{y} \beta_{m-1}^{\varphi 1}(\pi R)-\partial_{y} \beta_{m-1}^{\varphi 1}(-\pi R)} c_{j}^{y} .
$$

New basis functions now satisfying the above two periodic boundary on $w(x, y, t)$ can be build as

$$
\beta_{j}^{\varphi 2}=\beta_{j}^{\varphi 1}-C_{j}^{2} \beta_{m-1}^{\varphi 1}(j=1,2,3, \ldots, m-2),
$$

where $C_{j}^{2}=\left[\partial_{y} \beta_{j}^{\varphi 1}(\pi R)-\partial_{y} \beta_{j}^{\varphi 1}(-\pi R)\right] /\left[\partial_{y} \beta_{m-1}^{\varphi 1}(\pi R)-\partial_{y} \beta_{m-1}^{\varphi 1}(-\pi R)\right]$.

Finally, prescribing condition $\partial_{y y}^{2} w(x,-\pi R)=\partial_{y y}^{2} w(x, \pi R)$ of Eq. (27) yields,

$$
\beta_{j}^{\varphi 3}=\beta_{j}^{\varphi 2}-C_{j}^{3} \beta_{m-2}^{\varphi 2}(j=1,2,3, \ldots, m-3),
$$

where $C_{j}^{3}=\left[\partial_{y y}^{2} \beta_{j}^{\varphi 2}(\pi R)-\partial_{y y}^{2} \beta_{j}^{\varphi 2}(-\pi R)\right] /\left[\partial_{y y}^{2} \beta_{m-2}^{\varphi 2}(\pi R)-\partial_{y y}^{2} \beta_{m-2}^{\varphi 2}(-\pi R)\right]$.

The Gaussian basis $\beta_{j}^{\varphi 3}$ in Eq. (B8) is the final one we were looking for. It fulfills all periodic conditions in the cylindrical shell circumferential direction. 


\section{References}

[1] S. Conlon, J. Fahnline, F. Semperlotti, Numerical analysis of the vibroacoustic properties of plates with embedded grids of acoustic black holes, J. Acoust. Soc. Am. 137 (2015) 447-457.

[2] H. Ji, J. Luo, J. Qiu, L. Cheng, Investigations on flexural wave propagation and attenuation in a modified one-dimensional acoustic black hole using a laser excitation technique, Mech. Syst. Signal Pr. 104 (2018) $19-35$.

[3] H. Li, C. Touzé, A. Pelat, F. Gautier, X. Kong, A vibro-impact acoustic black hole for passive damping of flexural beam vibrations, J. Sound Vib. 450 (2019) 28-46.

[4] W. Huang, H. Zhang, D. J. Inman, J. Qiu, C. E. Cesnik, H. Ji, Low reflection effect by 3D printed functionally graded acoustic black holes, J. Sound Vib. 450 (2019) 96-108.

[5] L. Tang, L. Cheng, Periodic plates with tunneled Acoustic-Black-Holes for directional band gap generation, Mech. Syst. Signal Pr. 133 (2019) 106257.

[6] L. Ma, L. Cheng, Sound radiation and transonic boundaries of a plate with an acoustic black hole, J. Acoust. Soc. Am. 145 (2019) 164-172.

[7] X. Li, Q. Ding, Sound radiation of a beam with a wedge-shaped edge embedding acoustic black hole feature, J. Sound Vib. 439 (2019) 287-299.

[8] L. Tang, L. Cheng, Impaired sound radiation in plates with periodic tunneled Acoustic Black Holes, Mech. Syst. Signal Pr. 135 (2020) 106410

[9] L. Zhao, S. Conlon, F. Semperlotti, Broadband energy harvesting using acoustic black hole structural tailoring, Smart Mater. Struct. 23 (2014) 065021.

[10] H. Ji, Y. Liang, J. Qiu, L. Cheng, Y. Wu, Enhancement of vibration based energy harvesting using compound acoustic black holes, Mech. Syst. Signal Pr. 132 (2019) 441-456.

[11] Y. Wang, J. Du, L. Cheng, Power flow and structural intensity analyses of Acoustic Black Hole beams, Mech. Syst. Signal Pr. 131 (2019) 538-553.

[12] P. Zeng, L. Zheng, J. Deng, A. Elsabbagh, S. Xiang, T. Yan, Y. Wu, Flexural wave concentration in tapered cylindrical beams and wedge-like rectangular beams with power-law thickness, J. Sound Vib. 452 (2019) 82-96.

[13] H. Zhu, F. Semperlotti, Phononic thin plates with embedded acoustic black holes, Phys. Rev. B 91 (2015) 104304.

[14] H. Zhu, F. Semperlotti, Two-dimensional structure-embedded acoustic lenses based on periodic acoustic black holes, J. Appl. Phys. 122 (2017) 065104.

[15] S. S. Ganti, T.-W. Liu, F. Semperlotti, Topological edge states in phononic plates with embedded acoustic black holes, J. Sound Vib. (2019) 115060.

[16] J. Y. Lee, W. Jeon, Vibration damping using a spiral acoustic black hole, J. Acoust. Soc. Am. 141 (2017) $1437-1445$.

[17] S. Park, M. Kim, W. Jeon, Experimental validation of vibration damping using an archimedean spiral acoustic black hole, J. Sound Vib. 459 (2019) 114838.

[18] L. Tang, L. Cheng, H. Ji, J. Qiu, Characterization of acoustic black hole effect using a one-dimensional fully-coupled and wavelet-decomposed semi-analytical model, J. Sound Vib. 374 (2016) 172-184.

[19] J. Deng, L. Zheng, P. Zeng, Y. Zuo, O. Guasch, Passive constrained viscoelastic layers to improve the efficiency of truncated acoustic black holes in beams, Mech. Syst. Signal Pr. 118 (2019) 461-476.

[20] L. Ma, S. Zhang, L. Cheng, A 2D Daubechies wavelet model on the vibration of rectangular plates containing strip indentations with a parabolic thickness profile, J. Sound Vib. 429 (2018) 130-146.

[21] J. Deng, L. Zheng, O. Guasch, H. Wu, P. Zeng, Y. Zuo, Gaussian expansion for the vibration analysis of plates with multiple acoustic black holes indentations, Mech. Syst. Signal Pr. 131 (2019) 317-334.

[22] J. Deng, O. Guasch, L. Zheng, Ring-shaped acoustic black holes for broadband vibration isolation in plates, J. Sound Vib. 458 (2019) 109-122.

[23] J. Deng, O. Guasch, L. Maxit, L. Zheng, Annular acoustic black holes to reduce propagative Bloch-Floquet flexural waves in periodically supported cylindrical shells, in: INTER-NOISE and NOISE-CON Congress and Conference Proceedings, volume 259 (9), pp. 206-212.

[24] J. Deng, O. Guasch, L. Maxit, L. Zheng, Reduction of Bloch-Floquet bending waves via annular acoustic black holes in periodically supported cylindrical shell structures, Appl. Acoust. 169 (2020) 107424.

[25] M. Mironov, Propagation of a flexural wave in a plate whose thickness decreases smoothly to zero in a finite interval, Sov. Phys. Acoust. 34 (1988) 318-319.

[26] V. Krylov, F. Tilman, Acoustic black holes for flexural waves as effective vibration dampers, J. Sound Vib. 274 (2004) 605-619.

[27] V. Denis, A. Pelat, F. Gautier, B. Elie, Modal overlap factor of a beam with an acoustic black hole termination, J. Sound Vib. 333 (2014) 2475-2488.

[28] V. Denis, F. Gautier, A. Pelat, J. Poittevin, Measurement and modelling of the reflection coefficient of an Acoustic Black Hole termination, J. Sound Vib. 349 (2015) 67-79.

[29] L. Tang, L. Cheng, Enhanced acoustic black hole effect in beams with a modified thickness profile and extended platform, J. Sound Vib. 391 (2017) 116-126

[30] J. J. Bayod, Experimental study of vibration damping in a modified elastic wedge of power-law profile, J. Vib. Acoust. 133 (2011) 061003.

[31] M. R. Shepherd, C. A. McCormick, S. C. Conlon, P. A. Feurtado, Modeling and optimization of acoustic black hole vibration absorbers, J. Acoust. Soc. Am. 141 (2017) 4034-4034.

[32] M. R. Shepherd, P. A. Feurtado, S. C. Conlon, Multi-objective optimization of acoustic black hole vibration absorbers, J. Acoust. Soc. Am. 140 (2016) EL227-EL230. 
[33] C. A. McCormick, M. R. Shepherd, Design optimization of three styles of acoustic black hole vibration absorbers, J. Acoust. Soc. Am. 145 (2019) 1823-1824.

[34] C. A. McCormick, M. R. Shepherd, Design optimization and performance comparison of three styles of one-dimensional acoustic black hole vibration absorbers, J. Sound Vib. 349 (2019) 115164

[35] L. Ma, L. Cheng, Topological optimization of damping layout for minimized sound radiation of an acoustic black hole plate, J. Sound Vib. 458 (2019) 349-364.

[36] V. V. Krylov, A new type of vibration damper based on flexural wave propagation in laminated wedges of power-law profile, J. Acoust. Soc. Am. 110 (2001) 2654-2654.

[37] W. Huang, H. Ji, J. Qiu, L. Cheng, Analysis of ray trajectories of flexural waves propagating over generalized acoustic black hole indentations, J. Sound Vib. 417 (2018) 216-226.

[38] O. Guasch, M. Arnela, P. Sánchez-Martín, Transfer matrices to characterize linear and quadratic acoustic black holes in duct terminations, J. Sound Vib. 395 (2017) 65-79.

[39] N. Jiménez, V. Romero-García, V. Pagneux, J.-P. Groby, Rainbow-trapping absorbers: Broadband, perfect and asymmetric sound absorption by subwavelength panels for transmission problems, Sci. Rep. 7 (2017) 13595.

[40] O. Guasch, P. Sánchez-Martín, D. Ghilardi, Application of the transfer matrix approximation for wave propagation in a metafluid representing an acoustic black hole duct termination, Appl. Math. Model. 77 (2020) 1881-1893.

[41] J. P. Hollkamp, F. Semperlotti, Application of fractional order operators to the simulation of ducts with acoustic black hole terminations, J. Sound Vib. (2019) 115035.

[42] A. Karlos, S. J. Elliott, J. Cheer, Higher-order WKB analysis of reflection from tapered elastic wedges, J. Sound Vib. 449 (2019) 368-388.

[43] J. Y. Lee, W. Jeon, Exact solution of Euler-Bernoulli equation for acoustic black holes via generalized hypergeometric differential equation, J. Sound Vib. 452 (2019) 191-204.

[44] D. O'Boy, V. Krylov, Vibration of a rectangular plate with a central power-law profiled groove by the Rayleigh-Ritz method, Appl. Acoust. 104 (2016) 24-32.

[45] B. Sivasubramonian, G. Rao, A. Krishnan, Free vibration of longitudinally stiffened curved panels with cutout, J. Sound Vib. 226 (1999) 41-55.

[46] H. Shi, T. Yang, S. Jiang, W. Li, Z. Liu, Curvature effects on the vibration characteristics of doubly curved shallow shells with general elastic edge restraints, Shock Vib. 2015 (2015).

[47] L. Tang, L. Cheng, Broadband locally resonant band gaps in periodic beam structures with embedded acoustic black holes, J. Appl. Phys. 121 (2017) 194901.

[48] A. E. H. Love, A treatise on the mathematical theory of elasticity, Cambridge university press, 2013.

[49] L. Ma, L. Cheng, Numerical and experimental benchmark solutions on vibration and sound radiation of an Acoustic Black Hole plate, Appl. Acoust. 163 (2020) 107223.

[50] J. Deng, O. Guasch, L. Zheng, A semi-analytical method for characterizing vibrations in circular beams with embedded acoustic black holes, J. Sound Vib. (2020) 115307.

[51] Z. Kang, X. Zhang, S. Jiang, G. Cheng, On topology optimization of damping layer in shell structures under harmonic excitations, Struct. Multidiscip. Optim. 46 (2012) 51-67. 